\title{
Vertical Velocity Dynamics and Mixing in an Anticyclone near the Canary Islands
}

\author{
S. N. EstradA-Allis, ${ }^{a}$ B. BARCElÓ-Llull, ${ }^{b}$ E. PAllàs-SAnZ, ${ }^{a}$ A. RodrÍGueZ-SANTANA, ${ }^{c}$ \\ J. M. A. C. SOUZA, ${ }^{a}$ E. MASON,${ }^{\mathrm{d}, \mathrm{b}}$ J. C. MCWILLIAMS, ${ }^{\mathrm{e}}$ AND P. SANGRÀ,${ }^{\mathrm{c}, \mathrm{f}}$ \\ ${ }^{a}$ Centro de Investigación Científica y Educación Superior de Ensenada, Baja California, Mexico \\ ${ }^{\mathrm{b}}$ Mediterranean Institute for Advanced Studies (IMEDEA), Esporles, Spain \\ ${ }^{\mathrm{c}}$ Departamento de Física, Facultad de Ciencias del Mar, Universidad de Las Palmas de Gran Canaria, Las Palmas, Spain \\ ${ }^{\mathrm{d}}$ Applied Physics Laboratory, University of Washington, Seattle, Washington \\ e Department of Atmospheric and Oceanic Sciences, University of California, Los Angeles, Los Angeles, California
}

(Manuscript received 10 August 2017, in final form 18 September 2018)

\begin{abstract}
The complex structure of the vertical velocity field inside an anticyclonic eddy located just south of the Canary Islands is analyzed through a high-resolution ocean model. Based on the flow divergence, vertical velocity is decomposed into various forcing components. The analysis reveals that advection and stretching of vorticity are the most important forcing contributions to the vertical velocity within the eddy. In the mixed layer, a small-scale multipolar vertical velocity pattern dominates. This is the result of vertical mixing effects that enhance the surface vertical velocity by increasing the ageostrophic velocity profile. As a result, an ageostrophic secondary circulation arises that acts to restore thermal-wind balance, inducing strong vertical motions. Nonlinear Ekman pumping/ suction patterns resemble the small-scale vertical velocity field, suggesting that nonlinear Ekman effects are important in explaining the complex vertical velocity, despite an overestimate of its magnitude. In the eddy thermocline, the vertical velocity is characterized by a dipolar pattern, which experiences changes in intensity and axisymmetrization with time. The dipolar vertical velocity distribution arises from the imbalance between the advection and stretching of the vorticity forcing terms. A vertical velocity dipole is also obtained by solving a generalized omega equation from density and horizontal velocity fields, which also shows a preponderance of the ageostrophic term. The ubiquity of dipolar vertical velocity distributions inside isolated anticyclones is supported by recent observational findings in the same oceanic region.
\end{abstract}

\section{Introduction}

Knowledge of eddy dynamics, vertical mixing, horizontal advection, and vertical eddy fluxes is continually growing, driven in part by their impact on biogeochemical cycles and potential roles in the modulation of the global climate system (e.g., McGillicuddy et al. 2007; Mahadevan et al. 2008; Ponte et al. 2013; Sheen et al. 2014). In particular, the analysis of eddy vertical velocities and their relation to vertical mixing often represents a challenge due to the short spatial and temporal scales involved in their dynamics. This requires three-dimensional highresolution synoptic oceanographic observations. In this regard, numerical models are useful tools for understanding the production of vertical velocity at different spatiotemporal scales.

\footnotetext{
${ }^{\mathrm{f}}$ Deceased.
}

Corresponding author: S. N. Estrada-Allis, sheila@cicese.mx
Vertical velocity $w$ inside eddies is related to a variety of processes such as advection of vorticity (Hoskins et al. 1978; Viúdez et al. 1996; Pallàs-Sanz and Viúdez 2005), eddy-wind interaction (e.g., Martin and Richards 2001), linear and nonlinear Ekman effects (e.g., Thomas and Lee 2005; McGillicuddy et al. 2007; Mahadevan et al. 2008; Calil and Richards 2010), and current- and SSTinduced Ekman pumping (Gaube et al. 2015). Moreover, vertical velocity within eddies has a complex structure (e.g., Viúdez and Dritschel 2003; Koszalka et al. 2009; Calil and Richards 2010; Cardona and Bracco 2012; Nardelli 2013; Ponte et al. 2013; Barceló-Llull et al. 2017). The mechanisms that explain such structures within anticyclonic eddies are still not well understood. For instance, Viúdez and Dritschel (2003) show a quadrupolar pattern of vertical velocity in an inviscid, $f$-plane, nonforced anticyclonic elliptical vortex model. On the other hand, eddy-wind interaction may lead to an upwelling (downwelling) at the center of anticyclonic (cyclonic) eddies as shown by Martin and Richards (2001) and 

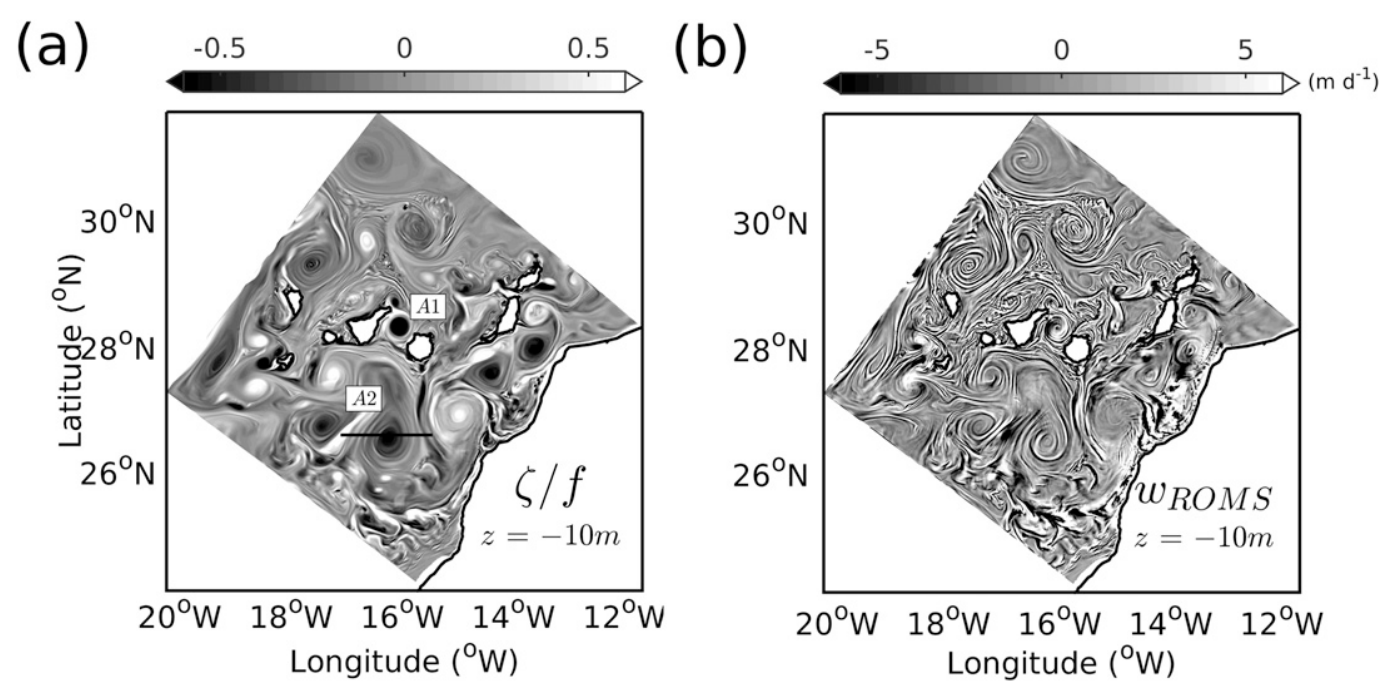

FIG. 1. Horizontal fields of the simulated 29 Aug. (a) Vertical component of relative vorticity normalized by the Coriolis parameter Ro $=\zeta / f$ at $z=-10 \mathrm{~m}$ and (b) vertical velocity $w_{\mathrm{ROMS}}\left(\mathrm{m} \mathrm{day}^{-1}\right)$ at $z=-10-\mathrm{m}$ depth. The two vortices analyzed in this study, A1 and A2, are indicated in (a). The black horizontal line indicates the zonal section.

McGillicuddy et al. (2007). This interaction generates a dipolar structure of vertical velocity due to nonlinear Ekman pumping (Stern 1965; Mahadevan et al. 2008; McGillicuddy et al. 2008). Moreover, wavelike vertical velocity patterns inside coherent eddies with alternating submesoscale cells of upwelling and downwelling are reported in the idealized primitive equation model of Koszalka et al. (2009). Through combined satellite and in situ observations, Nardelli (2013) reports a complex vertical velocity pattern for an Agulhas ring, likely due to the presence of vortex Rossby waves. Recently, Barceló-Llull et al. (2017) use high-resolution in situ observations from the so-called Canary Eddy Corridor (CEC; Sangrà et al. 2009) near the Canary Islands to resolve a generalized omega equation. They find a dipolar vertical velocity structure that is associated with an intrathermocline eddy. A dipolar vertical velocity is also investigated by Viúdez (2017) inside an isolated marginally spheroidal vortex. Viúdez (2017) suggests that the vertical velocity dipole is due to the precession of almost plane isopycnals, related to the tilting of the vortex.

Vertical mixing in the upper layers can also make an essential contribution to the vertical velocity structure. However, only a few studies have emphasized the effects of mixing on vertical motions. This is partly due to noise propagation through higher-order derivatives, nonlinear effects, and the significant dependence on mixing parameterizations that must be included in the general formulations. Garrett and Loder (1981) suggested that diabatic mixing can drive ageostrophic secondary circulation at fronts. Nagai et al. (2006) solved the quasigeostrophic (QG) and semigeostrophic (SG) diabatic equations (omega equation), showing that vertical mixing enhances vertical velocity in the upper ocean layers. PallàsSanz et al. (2010) used a $K$-profile parameterization (Large et al. 1994) of vertical mixing in a generalized omega equation for a California Current System front, and showed that the effects of mixing are constrained to the upper $100 \mathrm{~m}$, leading to an intensification of vertical velocity (Giordani et al. 2006; Johnston et al. 2011). Ponte et al. (2013) suggested that surface vertical mixing within the mixed layer tends to destroy thermal-wind balance, leading to the development of ageostrophic secondary circulation. However, their formulation is only valid at low Rossby number regimes, and they use a constant value for the diapycnal diffusivity coefficient $K_{v}$ (e.g., Gula et al. 2014). The turbulent thermal-wind (TTW) balance might also explain the upper ageostrophic secondary circulation associated with mesoscale structures, but especially at submesoscale flows with high Rossby numbers (McWilliams et al. 2015). However, the TTW balance is confined to the surface layer, where the eddy viscosity is large.

This study focuses on 1) analyzing the three-dimensional structure of the mesoscale vertical velocity inside two simulated anticyclonic eddies, a young circular anticyclone generated between Tenerife and Gran Canaria islands (denoted A1) and an open ocean, mature, elliptical anticyclone (denoted A2) shown in Fig. 1a; and 2) diagnosing the influence of diapycnal mixing on the vertical velocity field. The vortices analyzed here are 
generated by the perturbation of the Canary Current (CC) by the Canary Islands (Arístegui et al. 1994; Sangrà et al. 2005; Jiménez et al. 2008; Piedeleu et al. 2009; Mason et al. 2011) within a previously validated highresolution regional numerical model simulation (Mason 2009). The CEC is a major pathway for long-lived eddies in the northeast subtropical Atlantic. It represents an important source of oceanic eddy kinetic energy in this region, such as occurs in areas of strong eddying flows (Calil and Richards 2010; Sheen et al. 2014). Sangrà et al. (2009) observed a clear dominance of anticyclones over cyclones. Such dominance has been reported in several theoretical, observational, and modeling studies (e.g., Graves et al. 2006; Koszalka et al. 2009, 2010; Mason et al. 2014).

\section{Methods}

\section{a. Numerical model (ROMS)}

A numerical simulation with the primitive equation Regional Oceanic Modeling System (ROMS) is used to investigate the vertical velocity within two mesoscale eddies located in the lee of the Canary Islands. ROMS uses orthogonal curvilinear coordinates in the horizontal and terrain-following $s$ coordinates in the vertical (Haidvogel and Beckmann 1999). The barotropic and baroclinic momentum equations are resolved separately. The density is calculated diagnostically using the equation of state of seawater. For the purpose of the present study, it is important to emphasize that the vertical velocity is inferred from the horizontal divergence of the modeled currents. The ROMS outputs used here are obtained using the University of California, Los Angeles (UCLA) variant of the ROMS kernel (Shchepetkin and McWilliams 2005, 2009), and the model configuration is described in Mason (2009).

The numerical grid horizontal resolution is $\sim 1 \mathrm{~km}$, with 60 sigma layers in the vertical, hereinafter called region L2 (Fig. 1). This resolution is adequate to study mesoscale and associated submesoscale structures (Capet et al. 2008; Cardona and Bracco 2012) inside anticyclonic eddies of the CEC. A two-stage one-way nesting is used to achieve the desired L2 resolution over the Canary Islands. An intermediate $3-\mathrm{km}$ grid is used to couple the L2 domain with a parent model grid of $\Delta x=\Delta y=7.5 \mathrm{~km}$. Details of the model initial conditions and surface and lateral boundary forcing (all climatological), its validation, and further model characteristics can be found in Mason (2009) and Mason et al. (2010, 2011, 2012). L2 is integrated for three years, with daily averaged outputs of the threedimensional velocity field, salinity, temperature, and turbulent diapycnal coefficients derived from the $K$-profiling
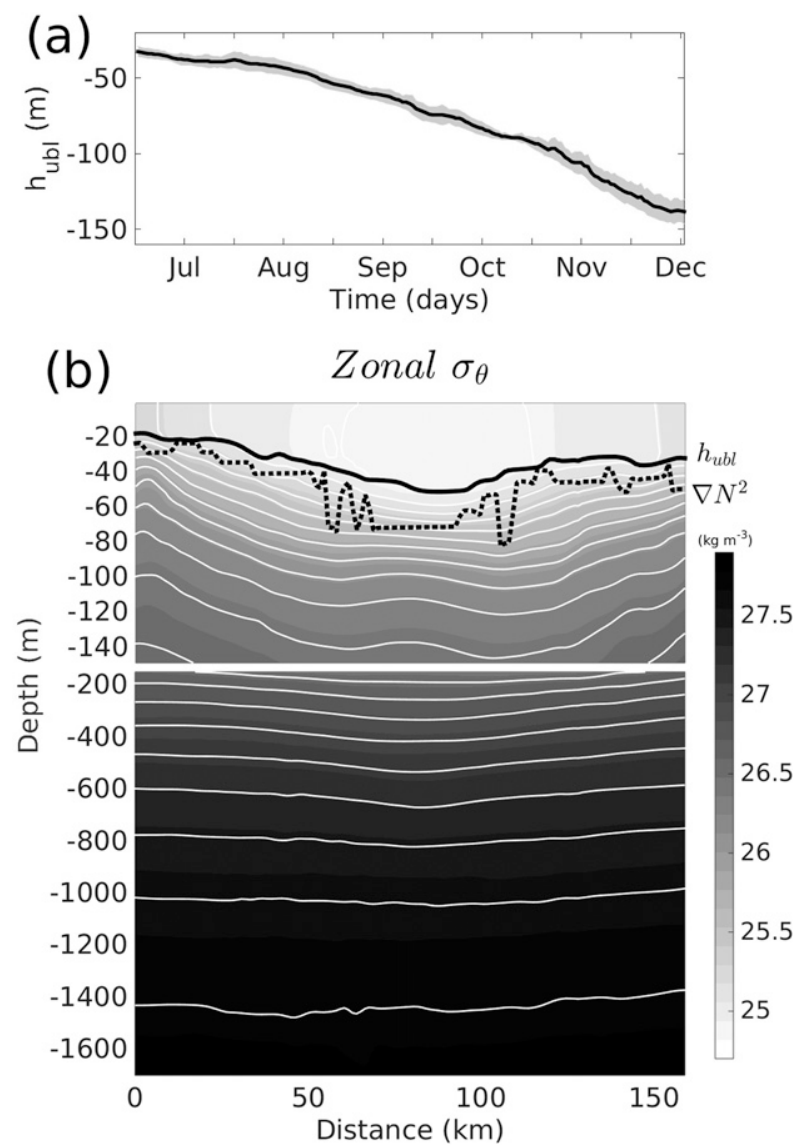

FIG. 2. (a) Upper boundary layer depth $h_{\mathrm{ubl}}$ (m) temporal series, from 15 Jul to $30 \mathrm{Dec}$, spatially averaged over the A2 eddy. (b) Zonal vertical section of potential density anomaly $\sigma_{\theta}\left(\mathrm{kg} \mathrm{m}^{-3}\right)$ from $15.4^{\circ}$ to $17^{\circ} \mathrm{W}$, at a fixed latitude of $26.55^{\circ} \mathrm{N}$, denoted in Fig. 1a as a black line, for the A2 vortex (29 Aug). White contours are isopycnals plotted every $0.15 \mathrm{~kg} \mathrm{~m}^{-3}$. The continuous black line is $h_{\text {ubl }}(\mathrm{m})$. The dashed line shows the surface maximum gradient of the square of the buoyancy frequency $N^{2}\left(\mathrm{~s}^{-2}\right)$.

parameterization (KPP) from Large et al. (1994). The model vertical velocity will be referred to as $w_{\text {ROMS }}$.

\section{b. Decomposition of the vertical velocity field by the flux divergence}

To examine the different contributions to the complex vertical velocity field of the A2 simulated eddy (Figs. 1 and 2), we infer the vertical velocity equation following the approach suggested by Vallis (2006) and implemented by Koszalka et al. (2009), based on the instantaneous horizontal divergence of the flux. This is in essence how ROMS computes the vertical velocity, and has been tested in similar numerical studies (Koszalka et al. 2009, 2010; Zhong et al. 2012). The main differences of our model in comparison with the idealized configuration of Koszalka et al. (2009) are in the atmospheric forcing 
term, due to the vertical distribution of the wind stress curl in the Ekman layer and the implementation of surface heat and water flux climatologies. The presence of rough bathymetry and topographic obstacles to the flow (i.e., the Canary Islands and the African coast) also contribute to a more realistic eddying regional ocean.

Following Vallis (2006) and Koszalka et al. (2009), the $w_{\text {ROMS }}$ field is examined through its contributions, as follows:

$$
\begin{aligned}
& w_{\mathrm{ROMS}}(x, y, z)=\frac{D \eta}{D t}+ \\
& \underbrace{-\int_{z}^{\eta}\left[\frac{1}{\left(f+\zeta_{1}\right)} \frac{D \zeta_{1}}{D t}\right] d z-\int_{z}^{\eta}\left[\frac{1}{\left(f+\zeta_{2}\right)} \frac{D \zeta_{2}}{D t}\right] d z}_{w_{\mathrm{Ageo}}} \\
& \underbrace{-\int_{z}^{\eta}\left[\frac{1}{\left(f+\zeta_{1}\right)}\left(\frac{\partial v}{D t} \zeta_{1}\right)\right] d z-\int_{z}^{\eta}\left[\frac{1}{\left(f+\zeta_{2}\right)} \frac{\partial u}{D t} \zeta_{2}\right] d z}_{w_{\mathrm{Str}}} \\
& \underbrace{-\int_{z}^{\eta}\left[\frac{1}{\left(f+\zeta_{1}\right)}\left(\frac{\partial w}{\partial x} \frac{\partial v}{\partial z}\right)\right] d z+\int_{z}^{\eta}\left[\frac{1}{\left(f+\zeta_{2}\right)}\left(\frac{\partial w}{\partial y} \frac{\partial u}{\partial z}\right)\right] d z}_{w_{\text {Tilt }}} \\
& \underbrace{+\int_{z}^{\eta}\left[A_{H} \frac{1}{\left(f+\zeta_{1}\right)}\left(\frac{\partial^{4} \zeta_{1}}{\partial x^{4}}+\frac{\partial^{4} \zeta_{1}}{\partial y^{4}}\right)\right] d z+\int_{z}^{\eta}\left[A_{H} \frac{1}{\left(f+\zeta_{2}\right)}\left(\frac{\partial^{4} \zeta_{2}}{\partial x^{4}}+\frac{\partial^{4} \zeta_{2}}{\partial y^{4}}\right)\right] d z}_{w_{\text {Hmix }}} \\
& \underbrace{+\int_{z}^{\eta}\left[\frac{1}{\left(f+\zeta_{1}\right)} \frac{\partial}{\partial x} \frac{\partial}{\partial z}\left(K_{v} \frac{\partial v}{\partial z}\right)\right] d z-\int_{z}^{\eta}\left[\frac{1}{\left(f+\zeta_{2}\right)} \frac{\partial}{\partial y} \frac{\partial}{\partial z}\left(K_{v} \frac{\partial u}{\partial z}\right)\right] d z}_{w_{\text {Vmix }}}
\end{aligned}
$$

where $u$ and $v$ are the zonal and meridional components of the velocity field; $\partial / \partial x, \partial / \partial y$, and $\partial / \partial z$ are the partial derivatives along the zonal, meridional, and vertical directions; $D / D t=\partial / \partial t+u \partial / \partial x+v \partial / \partial y+w \partial / \partial z$ denotes the material derivative; $\eta$ is the sea surface elevation; and $A_{H}$ is the constant biharmonic horizontal diffusion with a constant value of $10^{6} \mathrm{~m}^{4} \mathrm{~s}^{-1}$.

All terms are normalized by $\left(\zeta_{1}+f\right)^{-1}$ and $\left(\zeta_{2}+f\right)^{-1}$, where $\zeta_{1}=\partial v / \partial x, \zeta_{2}=-\partial u / \partial y$ and $\zeta=\zeta_{1}+\zeta_{2}$ is the vertical component of the relative vorticity. The sum of the rhs terms of Eq. (1) will be hereafter referred to as $w_{\text {sum. }}$. Differences between $w_{\text {ROMS }}$ and $w_{\text {sum }}$ are mainly due to offline computation of this equation, instead of computation at every time step as in ROMS. These differences can be seen in Figs. 3a, 3b, 4a, and 4b. As is done by ROMS, we take the wind stress $\tau$ as a surface boundary condition. Thereby, the wind effect in the vertical velocity field is explained in section 2c, in terms of a pumping Ekman mechanism, including the linear and nonlinear Ekman pumping in the Ekman layer. A full derivation of Eq. (1) is shown in the appendix.

The first rhs term of Eq. (1) formulates the material rate of change of $\eta$. The term $w_{\text {Ageo }}$ is the ageostrophic contribution and represents the material rate of change of the pseudo relative vorticity $\left(\zeta_{1}\right.$ or $\left.\zeta_{2}\right)$; that is, it represents the instantaneous vorticity changes and the advection of vorticity by ageostrophic effects (Koszalka et al. 2009, 2010; Zhong et al. 2012). The term $w_{\text {Str }}$ is the stretching of vorticity, and $w_{\text {Tilt }}$ is the vortex tilting. Through Eq. (1), Koszalka et al. (2009) and Zhong et al. (2012) show that the vertical velocity is very complex within the eddies. They associate this complexity with the interplay of advection, stretching and instantaneous vertical velocity changes. The last two rhs terms of Eq. (1) account for the influence of mixing on the vertical velocity field; these are the horizontal mixing $w_{\text {Hmix }}$ and vertical or diapycnal mixing $w_{\text {Vmix }}$ contributions.

The diagnostic of vertical velocity through Eq. (1) allows the inclusion of diabatic sources such as the vertical mixing, which can be analyzed in detail. Other forcing terms, such as total stretching and tilting terms, are not included in the QG approximation but are present in Eq. (1) (Koszalka et al. 2009). Therefore, Eq. (1) allows us to analyze all the individual forcing terms for the complex pattern of the vertical velocity within the simulated anticyclonic eddy. Furthermore, to 

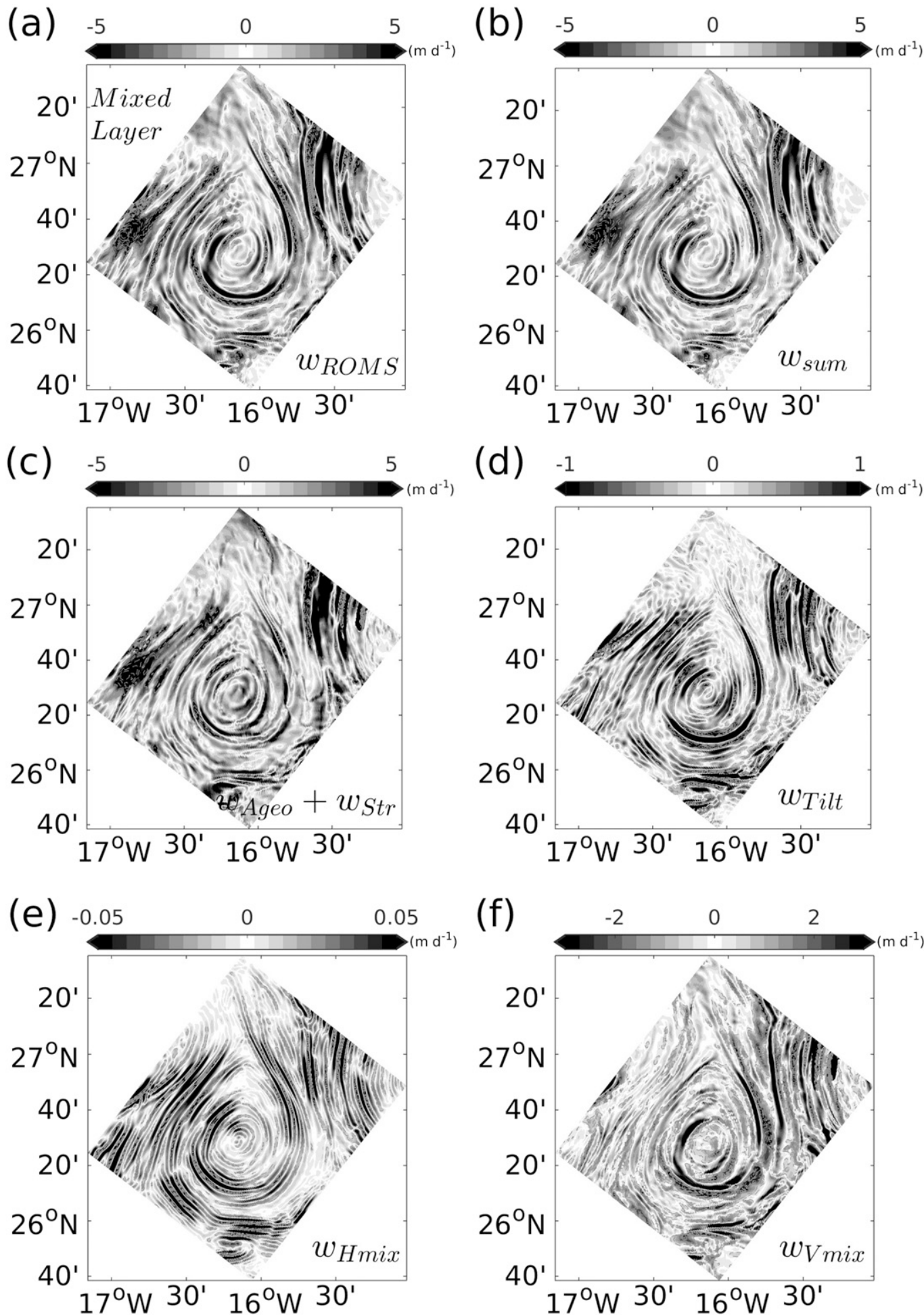

FIG. 3. Horizontal fields for 29 Aug averaged over $z=[-1,-20] \mathrm{m}$, corresponding to each contribution of the vertical velocity $\left(\mathrm{m} \mathrm{day}^{-1}\right)$. (a) Vertical velocity given by the ROMS model ( $\left.w_{\text {ROMS }}\right)$ and (b) the sum of the rhs terms in Eq. (1) $\left(w_{\text {sum }}\right),(\mathrm{c})$ the sum of ageostrophic $\left(w_{\text {Ageo }}\right)$ and stretching $\left(w_{\text {Str }}\right)$ contributions, (d) tilting contribution $\left(w_{\text {Tilt }}\right)$, (e) horizontal mixing contribution $\left(w_{\text {Hmix }}\right)$, and (f) vertical mixing contribution $\left(w_{\text {Vmix }}\right)$. Superimposed light gray contours denote negative values of vertical velocity. Note the changes in the scale of color bars. All fields have units of $\mathrm{mday}^{-1}$. 

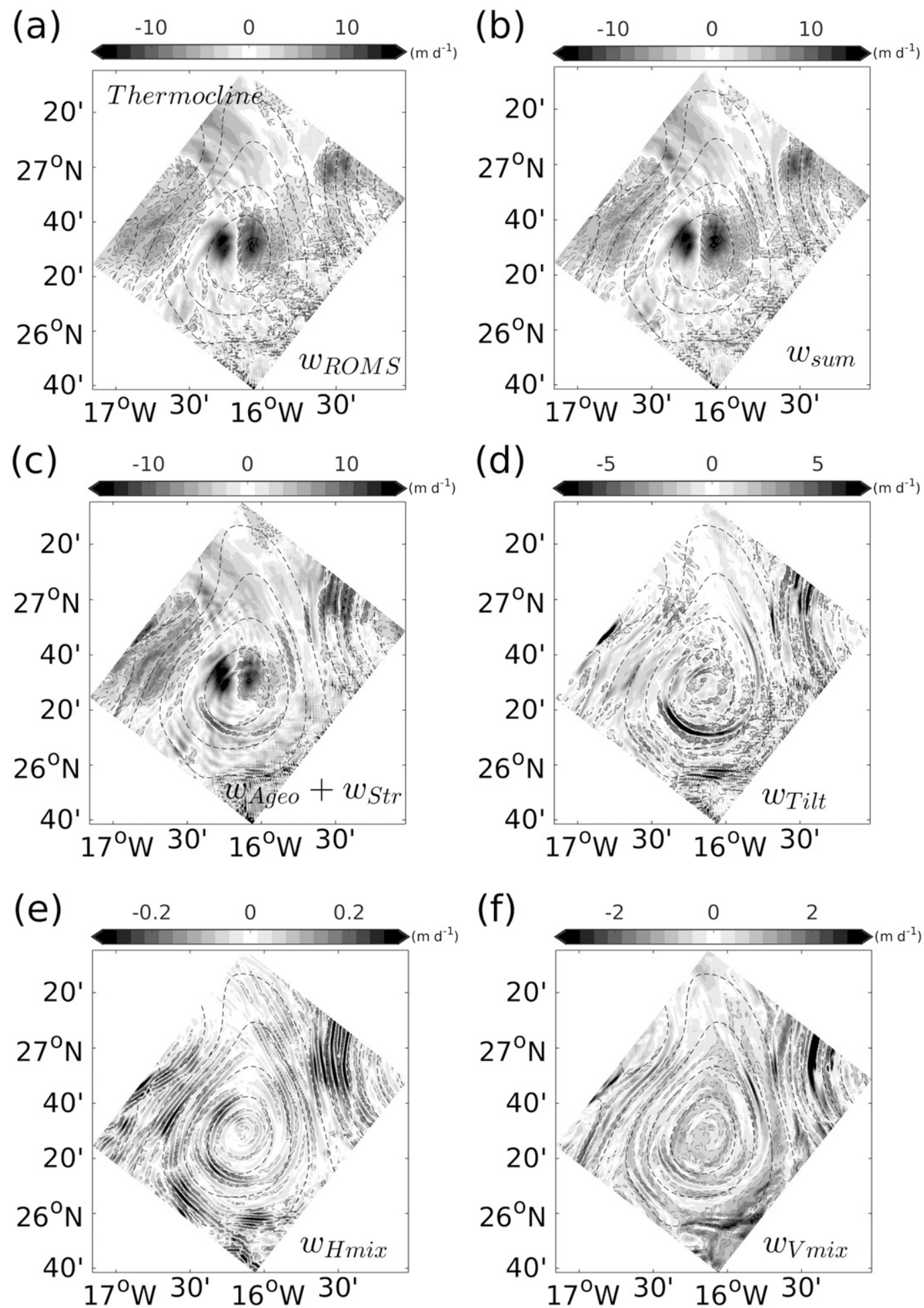

FIG. 4. As in Fig. 3, but averaged over $z=[-200,-800] \mathrm{m}$. Superimposed light gray contours denote negative values of vertical velocity. Note the changes in the color bars. Dashed black contours are the free surface elevation $\eta(\mathrm{m})$, to highlight the shape of the A2 eddy. 
better understand the dynamics of the vertical velocity, a diabatic generalized omega equation was used to diagnose the vertical velocity (e.g., Viúdez and Dritschel 2004; PallàsSanz and Viúdez 2005; Barceló-Llull et al. 2017), which can be solved for flows with high Rossby numbers by including the ageostrophic forcing terms (see section $2 \mathrm{~d}$ ).

Two depth ranges have been selected, $z \in$ [surface, $-20] \mathrm{m}$ (within the mixed layer) and $z \in[-100,-800] \mathrm{m}$ (within the thermocline). Although the analyses are focused on the A2 eddy (Fig. 1a), similar results using this model configuration are obtained for other anticyclonic eddies with similar Rossby number (Ro) and horizontal divergence fields ubiquitous in the CEC (not shown).

\section{c. Wind-induced vertical velocities}

The inclusion of the advection of vertical vorticity by the Ekman transport itself or so-called nonlinear Ekman pumping has important and recognized consequences for the development of vertical velocities inside eddies (see, e.g., Stern 1965). The main implication of nonlinear Ekman effects lies in that even a uniform (irrotational) wind stress field will lead to nonlinear Ekman vertical velocities. To explicitly examine the role of nonlinear and linear contributions of the wind-driven vertical velocities inside the anticyclone, we have computed the total Ekman pumping/suction velocity $w_{\text {EkT }}$, splitting the linear $w_{\mathrm{EkL}}$ and nonlinear $w_{\mathrm{EkNL}}$ contributions (Stern 1965):

$$
w_{\mathrm{EkT}}=w_{\mathrm{EkL}}+w_{\mathrm{EkNL}},
$$

where the linear contribution $w_{\mathrm{EkL}}$ is

$$
w_{\mathrm{EkL}}=\frac{1}{\rho_{o}\left(f+\zeta_{g}\right)}\left(\frac{\partial \tau^{y}}{\partial x}-\frac{\partial \tau^{x}}{\partial y}\right),
$$

with $\tau^{x}$ and $\tau^{y}$ being the components of the wind stress. This is the linear contribution where the wind curl induces a divergence (or convergence, depending on the wind-curl sign) of the Ekman transport and thus an upwelling (or downwelling) at the eddy center. The nonlinear term, $w_{\mathrm{EkNL}}$, is related to the advection of absolute vorticity $\left(f+\zeta_{g}\right)$ by the Ekman transport, where $\zeta_{g}$ is the surface geostrophic relative vorticity, such that $\zeta_{g}=\partial v_{g} / \partial x-\partial u_{g} / \partial y$, where $u_{g}$ and $v_{g}$ are the horizontal components of the geostrophic velocity, by the wind-induced Ekman transport in the mixed layer (e.g., Niiler 1969; Thomas and Rhines 2002; Mahadevan and Tandon 2006). It can be formulated as

$$
w_{\mathrm{EkNL}}=\frac{1}{\rho_{o}\left(f+\zeta_{g}\right)^{2}}\left[\tau^{x} \frac{\partial}{\partial y}\left(f+\zeta_{g}\right)-\tau^{y} \frac{\partial}{\partial x}\left(f+\zeta_{g}\right)\right],
$$

where $\rho_{o}=1026 \mathrm{~kg} \mathrm{~m}^{-3}$ is the mean density of water within the eddy.
Notice that Eq. (2) is the stationary definition of the surface Ekman layer dynamics over geostrophic flow. The estimation of the frictional vertical velocity inside the eddy is computed by considering sustained winds; in this particular area (trade) winds usually last longer than one inertial period. Furthermore, we force the model with monthly wind fields rather than daily.

The wind stress is parameterized in terms of the wind speed $10 \mathrm{~m}$ above the sea surface level $\left(\mathbf{U}_{10}\right)$ :

$$
\boldsymbol{\tau}=\rho_{a} C_{d} \mathbf{U}_{10}\left|\mathbf{U}_{10}\right|,
$$

where $\rho_{a}=1.22 \mathrm{~kg} \mathrm{~m}^{-3}$ is the air density. Here $C_{d}$ is the drag coefficient as a function of the wind velocity parameterized following Yelland et al. (1998) for low wind speeds $\left(3 \leq \mathbf{U}_{10} \leq 6 \mathrm{~m} \mathrm{~s}^{-1}\right)$ as

$$
1000 C_{d}=0.29+\frac{3.1}{\mathbf{U}_{10}}+\frac{7.7}{\mathbf{U}_{10}^{2}},
$$

whereas at high wind speeds $\left(6 \leq \mathbf{U}_{10} \leq 26 \mathrm{~m} \mathrm{~s}^{-1}\right)$

$$
1000 C_{d}=0.50+0.071 \mathbf{U}_{10} .
$$

\section{d. Decomposition of the vertical velocity field by a generalized omega equation}

To compare the vertical velocity obtained from the horizontal flux divergence (i.e., $w_{\text {ROMS }}$ ) with an independent estimation of mesoscale vertical velocity, we have solved the generalized omega equation from density and horizontal velocity (Viúdez et al. 1996; PallàsSanz and Viúdez 2005; Pallàs-Sanz et al. 2010) ( $w_{\text {OMEGA }}$ hereafter). In terms of the $\mathbf{Q}$ vector, the approximated generalized omega equation (Viúdez and Dritschel 2004) is expressed as follows:

$$
N^{2} \nabla_{h}^{2} w+f(f+\zeta) \frac{\partial^{2} w}{\partial z^{2}}=\underbrace{2 \nabla_{h} \cdot \mathbf{Q}_{h}}_{F_{\mathrm{OT}}}+\underbrace{f \boldsymbol{\zeta}_{p h}^{a} \cdot \nabla_{h}^{2} \mathbf{u}_{h}}_{F_{\mathrm{PSE}}},
$$

with $\mathbf{Q}_{h}$ being the generalized $\mathbf{Q}$ vector:

$$
\mathbf{Q}_{h}=\nabla_{h} \mathbf{u}_{h} \cdot \nabla_{h} \sigma_{\theta},
$$

where the subscript $h$ denotes horizontal components; $\zeta_{p h}^{a}=\left(-\partial_{z} v^{a}, \partial_{z} u^{a}\right)$ is the ageostrophic pseudovorticity vector and $\left(u^{a}, v^{a}\right)$ are the horizontal ageostrophic velocities. We refer to Viúdez et al. (1996) and Pallàs-Sanz and Viúdez (2005) for further details. Notice that in Eq. (8) the effects of diapycnal mixing are not included. Hence, results from this analysis will be presented only for the stratified fluid below the mixed layer (see Fig. 2).

The first rhs term in Eq. (8) can be seen as the horizontal divergence of the straining deformation by the 
total horizontal current field. The second rhs term is the ageostrophic pseudovorticity forcing term $F_{\mathrm{PSE}}$. One of the advantages of the generalized omega equation is that it explicitly includes both geostrophic and ageostrophic components of the horizontal velocity in the divergence of the $\mathbf{Q}_{h}$ vector (Viúdez et al. 1996; Pallàs-Sanz and Viúdez 2005), and hence it can be decomposed into the geostrophic component $\mathbf{Q}^{g}$ and the ageostrophic component $\mathbf{Q}^{a}$ such as the divergence of the geostrophic $\mathbf{Q}$ vector, $F_{\mathrm{QG}}=2 \nabla_{h} \cdot \mathbf{Q}_{h}^{g}$, and its ageostrophic counterpart $F_{\mathrm{QA}}=2 \nabla_{h} \cdot \mathbf{Q}_{h}^{a}$. Through the integration of each individual forcing term in Eq. (8), we obtain the vertical velocity contributions of the deformation field $w_{\mathrm{QT}}$, the contribution of the pseudovorticity term $w_{\mathrm{PSE}}$ and, with the decomposition of the deformation $\mathbf{Q}_{h}$ vector in its geostrophic and ageostrophic counterparts, we obtain the mesoscale vertical velocity driven by purely geostrophic $w_{\mathrm{QG}}$ and ageostrophic deformation $w_{\mathrm{QA}}$. Consequently, $w_{\mathrm{OMEGA}}=w_{\mathrm{QT}}+w_{\mathrm{PSE}}$ and $w_{\mathrm{QT}}=w_{\mathrm{QG}}+w_{\mathrm{QA}}$.

\section{Results}

The model reproduces the rich mesoscale variability observed south of the Canary Islands in the form of anticyclonic and cyclonic mesoscale eddies (Fig. 1a). Notice that submesoscale structures associated with mesoscale eddy interactions, such as small-scale filaments, arise in the high-resolution simulations (Lapeyre and Klein 2006; Capet et al. 2008). The eddy field is characterized by high Rossby numbers Ro $=\zeta / f$. Mean values of $|R o=0.7|$ near the surface indicate that ageostrophy and, thus, vertical velocities may be significant. Local maxima of $|\mathrm{Ro}| \simeq 3.5$ are associated with submesoscale structures such as small-scale eddies and filaments. Values of $|\mathrm{Ro}| \simeq 5$ have been recently obtained using drifter observations associated to submesoscale fronts in the Southern California Bight (Ohlmann et al. 2017). Complex horizontal distribution of the vertical velocity associated with the periphery of mesoscale eddies is observed (Fig. 1b). At these depths $(z=-10 \mathrm{~m})$ mesoscale vertical velocity is masked by enhanced filamentary distributions of vertical velocity at the eddy peripheries resembling submesoscale fronts.

Two anticyclonic eddies, A1 and A2, at a specific date (29 August), have been selected for analysis of the vertical velocity distribution (Fig. 1a), a young circular anticyclone generated between Tenerife and Gran Canaria islands (A1) and an open ocean, mature, elliptical anticyclone (A2). The A2 vortex has relatively high $|\mathrm{Ro}|$ values with strong negative $\zeta$ at the eddy core $(\operatorname{Ro}=-0.7)$. Frontal filaments with alternating signs of $\zeta$ are visible between the eddies as a result of straining during eddyeddy interactions.
The vertical density structure of the A2 vortex is shown in Fig. 2. As expected for anticyclonic eddies, the isopycnals deepen at the eddy center down to $z=-800 \mathrm{~m}$ where they are approximately flat, indicating the base of the eddy. At the eddy periphery the isopycnals outcrop at the surface. This eddy has an elliptical shape with a mean radius of $40.85 \pm 2.58 \mathrm{~km}$ and experiences changes in its aspect ratio as it propagates westward along the CEC. Previous observations of westward propagating of $\mathrm{CEC}$ eddies by Sangrà et al. (2009) suggested that these eddies modify their aspect ratio during their translation. The radius and aspect ratio are obtained by wavelet analysis of the Lagrangian trajectories of virtual drifters released inside the A2 eddy (not shown). The deepening of the isopycnals at the eddy center increases the mixed layer depth, computed using the KPP, which usually refers to the upper boundary layer $h_{\mathrm{ubl}}$ (Fig. 2a and continuous line in Fig. 2b). This results in high stratification below $h_{\mathrm{ubl}}$, delimited by the maximum of the squared buoyancy frequency $N^{2}$ (dashed line in Fig. 2). The simulated $h_{\mathrm{ubl}}$ has an annual mean value of $h_{\mathrm{ubl}}=65.22 \pm 29.88 \mathrm{~m}$, averaged over the whole numerical L2 domain. In particular, the A2 eddy has, on average, $h_{\mathrm{ubl}}=35 \mathrm{~m}$ at the edges and $h_{\mathrm{ubl}}=80 \mathrm{~m}$ in the center of the eddy. Interestingly, the averaged $h_{\mathrm{ubl}}$ of the A2 eddy deepens as the eddy evolves (Fig. 2a).

\section{a. Forcing of the eddy vertical velocity}

In the mixed layer, a snapshot of the $w_{\text {ROMS }}$ field reveals a complex submesoscale wavelike pattern (Fig. 3a) in which elongated cells or filaments of downwelling and upwelling alternate around the vortex core. Results also show that vertical velocities are more intense at the eddy periphery. The sum of the rhs terms of Eq. (1) is in agreement with $w_{\text {ROMS }}$, indicating that the vertical velocity budget is in balance (Figs. 3a,b and $4 a, b)$.

Decomposition of $w_{\text {ROMS }}$ by Eq. (1) shows that $w_{\text {Ageo }}$ and $w_{\text {Str }}$ are the most important contributions to $w_{\text {ROMS }}$, driving the vertical velocity distribution in the mixed layer (Fig. 3c). Both exhibit the shape of an azimuthal wave with a wavenumber of order 4 , but they are out of phase, thus largely canceling each other (not shown) and so being represented here as a sum. The term $w_{\text {Vmix }}$ is the second most important contribution to vertical velocity with maximum values of $\pm 3 \mathrm{~m}_{\text {day }}{ }^{-1}$ (Fig. 3f).

The wavelike pattern with alternating elongated cells of vertical velocity in $w_{\text {Tilt }}, w_{\text {Hmix }}$, and $w_{\text {Vmix }}$ (Figs. 3d-f) seems to match the spiral structure of the $w_{\text {ROMS }}$ pattern. However, both $w_{\text {Tilt }}$ and $w_{\text {Hmix }}$ have magnitudes from 5 to 100 times smaller than the overall $w_{\text {ROMS }}$. Vertical velocities due to $\eta$ [first term on the rhs of Eq. (1)] are 

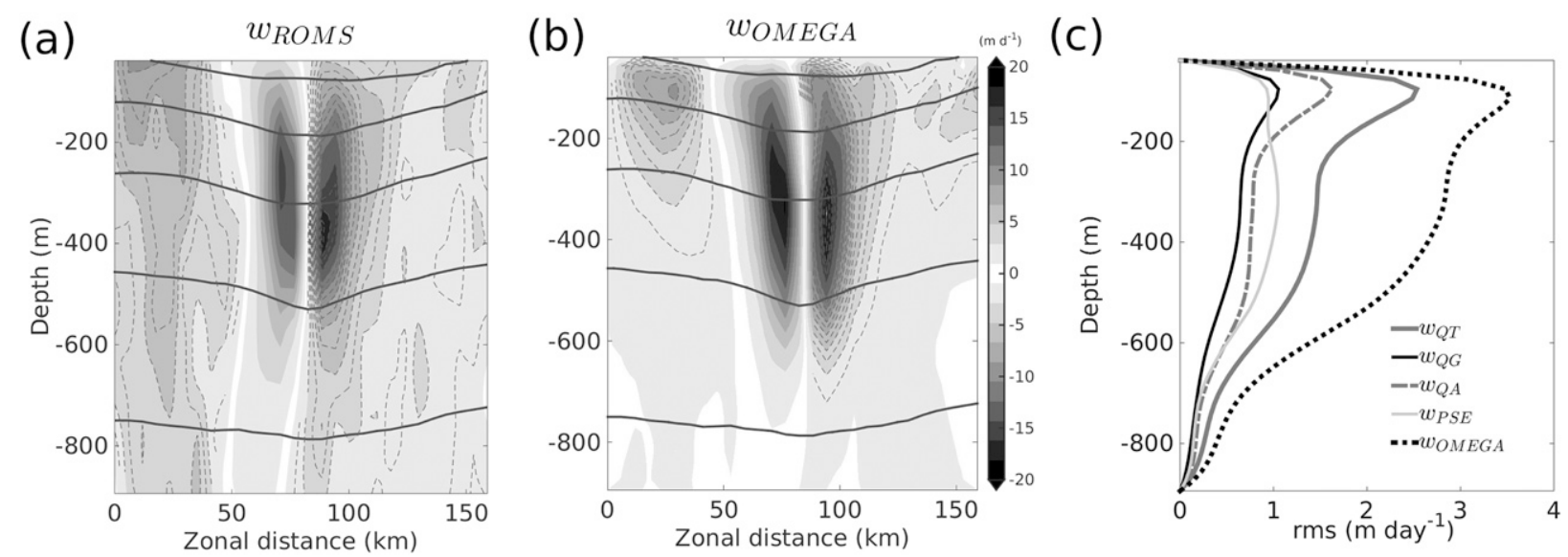

FIG. 5. Zonal vertical sections for the A2 eddy of (a) total vertical velocity from the model $w_{\text {ROMs }}$, and (b) total vertical velocity computed with all the forcing terms in the right-hand side of Eq. (8) $\left(F_{\mathrm{QT}}+F_{\mathrm{PSE}}\right), w_{\mathrm{OMEGA}}\left(\mathrm{m} \mathrm{day}^{-1}\right)$. Gray contours are isopycnals plotted every $0.25 \mathrm{~kg} \mathrm{~m}^{-3}$. Dashed gray contours denote negative values of vertical velocity. Both vertical velocities sections have a horizontal resolution of $5 \mathrm{~km}$. (c) The root-mean-square of the vertical velocities estimated with each individual forcing term in Eq. (8).

on average one order of magnitude less than $w_{\text {ROMS }}$ (not shown).

In the thermocline, the instantaneous $w_{\text {ROMS }}$ field consists of a cell of upwelling-downwelling at the eddy core, differing from the $w_{\text {ROMS }}$ complex submesoscale mixed layer structure. The sum of $w_{\text {Ageo }}$ and $w_{\text {Str }}$ is again the most important contribution to $w_{\text {ROMS }}$ (Fig. 4c). Intense vertical velocity arises in places where $w_{\text {Ageo }}$ and $w_{\text {Str }}$ do not cancel completely each other. The remaining contributions act as secondary forcing for the vertical velocity eddy field within the thermocline (Figs. 4d-f). The upwelling and downwelling cell coincides with moderately high Ro values $(|\mathrm{Ro}|=0.3-0.5)$ around the eddy center and fades at the eddy base $(z=-800 \mathrm{~m}$; not shown).

\section{b. A comparison with a generalized omega equation}

Forcing terms of the omega equation are analyzed to obtain an alternative physical interpretation of the vertical velocity (Fig. 5c). The comparison between $w_{\text {ROMS }}$ and $w_{\text {OMEGA }}$ is made by decreasing the resolution of the model output fields (density and horizontal velocity) to $5 \mathrm{~km}$ in the horizontal and to $20 \mathrm{~m}$ in the vertical. The magnitude of the vertical velocity dipole of $w_{\mathrm{OMEGA}}$ is underestimated by an order of magnitude relative to $w_{\text {ROMS }}$ when Eq. (8) is forced with the original horizontal resolution of $\sim 1 \mathrm{~km}$, although the dipole of vertical velocity is still present. This can be attributed to high submesoscale variability of the forcing on the rhs of Eq. (8), which would introduce strong gradients in the elliptic equation. Hence, the relaxation method used to solve the equation would act to smooth the velocity field by successive iterations. In consequence, this study shows that higher-order versions of the omega equation need to be used as the model resolution is increased. With this, the comparison shows that $w_{\text {ROMS }}$ and $w_{\text {OMEGA }}$ are very similar (Figs. 5a,b). Both vertical velocity fields show a dipolar distribution of vertical velocity centered at the eddy center, with the maxima located at around 300-m depth for both fields.

As illustrated in Fig. 5c, the sum of $w_{\mathrm{QT}}$ and $w_{\mathrm{PSE}}$ will give the overall diagnosed vertical velocity $w_{\mathrm{OMEGA}}$. Note that $w_{\text {OMEGA }}$ is mainly induced by the total deformation of the density gradient $\left(w_{\mathrm{QT}}\right)$. Calil and Richards (2010) found that the divergence of the geostrophic $\mathbf{Q}$ vector was consistent with the simulated $w_{\text {ROMS }}$ for a mesoscale eddy near the Hawaiian coast, especially in regions of intense vertical flows. We find that a preponderance of the ageostrophic forcing is found at the eddy thermocline, where $w_{\mathrm{QA}}$ dominates over $w_{\mathrm{QG}}$. In the first $200 \mathrm{~m}$ of the water column $w_{\mathrm{QA}}$ is the main contributor to $w_{\text {OMEGA }}$. Below $200 \mathrm{~m}$ until the base of the eddy, $w_{\mathrm{PSE}}$ is the main contributor to the total $w_{\mathrm{OMEGA}}$.

\section{c. Temporal evolution of the vertical velocity contributions terms}

To demonstrate the temporal evolution of the rhs terms of Eq. (1), we show the evolution of the spatial root-mean-square for each contributing term, averaged over the mixed layer (Fig. 6a) and over the thermocline (Fig. 6b) are shown. The overall vertical velocity continues to be mainly dominated by the sum of $w_{\text {Ageo }}$ and $w_{\text {Str }}$ at all times. Near the surface $w_{\text {Ageo }}$ and $w_{\text {Str }}$ tend to increase with time from the end of August. There is a transition of the regime of the evolution of the forcing terms for vertical velocity after this date (hereafter, transition time), with $w_{\text {Vmix }}$ increasing with time along 

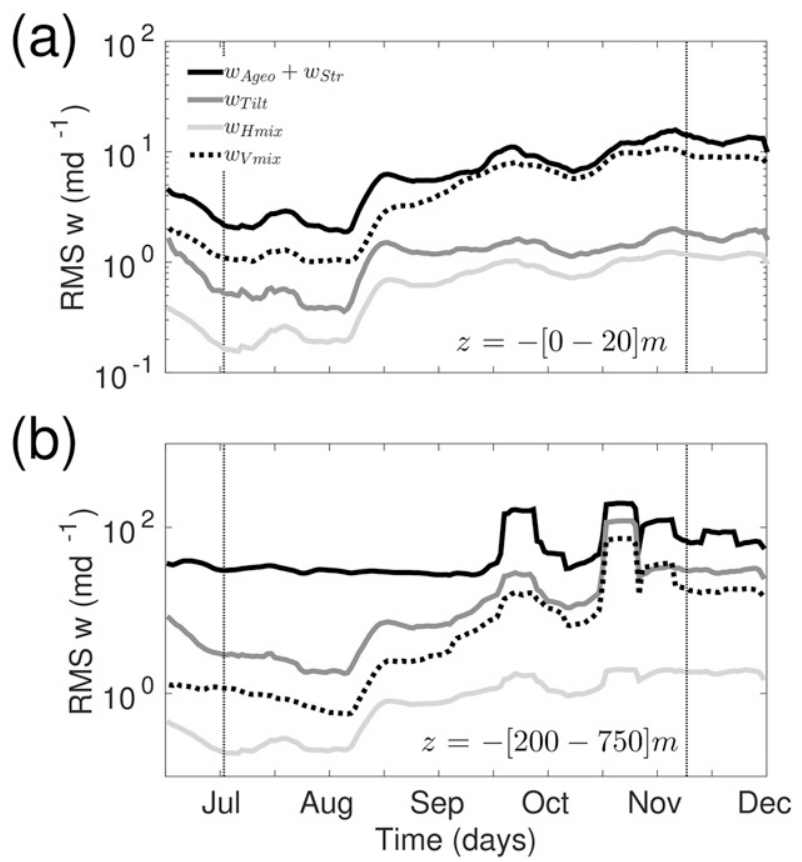

FIG. 6. Root-mean-square (rms) temporal evolution of each contribution term $\left(\mathrm{m} \mathrm{day}^{-1}\right)$ for (a) rms averaged over the mixed layer, $z=$ [surface, -20$] \mathrm{m}$, and (b) rms averaged over the pycnocline, $z=$ $[-200,-800] \mathrm{m}$ depth . The temporal series are smoothed by using weighted linear regression to improve visualization. Vertical dashed lines represent the dates used to compare two eddy stages; i.e., before and after the transition date in Fig. 7, for 1 Aug and 8 Dec, respectively. The $x$ axis denotes the end of each month.

with $w_{\text {Ageo }}$, and with deepening of the mixed layer depth (Fig. 2a). The remaining contribution terms also increase but keeping a secondary role as forcing terms to $w_{\text {ROMS }}$.

At the thermocline, the dominant forcing terms $w_{\text {Ageo }}$ and $w_{\text {Str }}$ slightly increase from the end of August, together with $w_{\text {Tilt }}$, which becomes a relevant forcing term for $w_{\text {ROMS }}$. This enhancement is accompanied by an increase of $w_{\text {Vmix }}$. Also, $w_{\text {Hmix }}$ is enhanced like the other contribution terms, although they still have a secondary order of importance related to $w_{\text {Ageo }}$ and $w_{\text {Str }}$.

Figure 7 shows horizontal and vertical sections of $w_{\text {ROMS }}$ inside the $\mathrm{A} 2$ eddy for two specific dates, before (10 August) and after (8 December) the transition time, at both mixed layer and thermocline eddy layers. The first case represents an early eddy life stage (Figs. 7a-c) whereas the second case shows a mature stage of the eddy (Figs. 7d-f). Concerning vertical velocity patterns, in the mixed layer, the small-scale vertical velocity cells are reinforced during winter. At the thermocline, small-scale structures are enhanced, and the dipole is more patchy. The upper layer vertical sections of each eddy stage (Figs. 7c,f) support the above picture, displaying a significant enhancement of $w_{\text {ROMS }}$ within the mixed layer toward winter times.
For the particular case of the A2 eddy the diapycnal mixing increases with time, this can be seen in Fig. 8 comparing two dates before and after the transition time. This increase is reflected in the increase of the vertical mixing contribution term $w_{\text {Vmix }}$ in Figs. $6 \mathrm{a}$ and $6 \mathrm{~b}$. A constant value of $K_{v}=10^{-5} \mathrm{~m}^{2} \mathrm{~s}^{-1}$ in the ocean interior is included in order to parameterize the effects of background turbulence, such as breaking of internal waves (Large et al. 1994). Only some eddies exhibit $K_{v}$ above the background value in the stratified thermocline, such as the case of the A2 eddy (Figs. 8b,d), which in turn also exhibits the highest values of $\mid$ Ro $\mid$ in its core (see Fig. 1a).

\section{d. The nonlinear Ekman effects in the eddy vertical velocity}

To analyze the effect of the wind in the vertical velocity patterns within the anticyclonic eddy, the total Ekman pumping [Eq. (2)] is compared for three different cases in Fig. 9. First, a uniform wind blowing over an analytical mesoscale Gaussian vortex is considered (Figs. 9a-c). Second, nonuniform trade winds, given by model outputs, blowing over the A1 eddy are explored (Figs. 9d-f). As the anticyclone A1 is at a young stage, it still retains its axisymmetric shape. Total Ekman pumping on the elliptical A2 eddy is computed using nonuniform trade winds (Figs. 9g-i). To keep consistent comparisons, Ro for the three eddies has the same order of magnitude (Figs. 9a,d,g).

Wind-driven vertical velocities for the Gaussian vortex lead to a dipolar distribution of the $w_{\text {EkT }}$ field consisting of an upwelling and a downwelling cell (Fig. 9c). The A1 vortex also leads to a dipolar distribution of the $w_{\text {EkT }}$ field, but it is clearly distorted with respect to the idealized configuration of the Gaussian vortex, with an increase of $w_{\text {EkT }}$ magnitude toward the eddy periphery where strong gradients of geostrophic vorticity occur (Fig. 9f). For the case of the $\mathrm{A} 2$ eddy, the pattern of the $w_{\mathrm{EkT}}$ field is much more complex, displaying important submesoscale variability (Fig. 9i), and resembling the wavelike vertical velocity pattern seen in the upper layers (see Fig. 3a). The $w_{\text {EkNL }}$ field (Figs. 10e,f) resembles the $w_{\text {EkT }}$ field (Figs. 9f,i), with downwelling on the western flank and upwelling on the eastern flank for both A1 and A2 eddies, indicating the dominance (2-3 times larger) of nonlinear Ekman pumping over the linear Ekman pumping (Figs. 10a-d).

\section{Discussion}

\section{a. Vertical mixing enhances vertical velocity at the mixed layer of the eddy}

Our results indicate that the influence of $w_{\text {Vmix }}$ forcing is constrained within the mixed layer (Nagai et al. 2006; Koszalka et al. 2010; Pallàs-Sanz et al. 2010), and may 

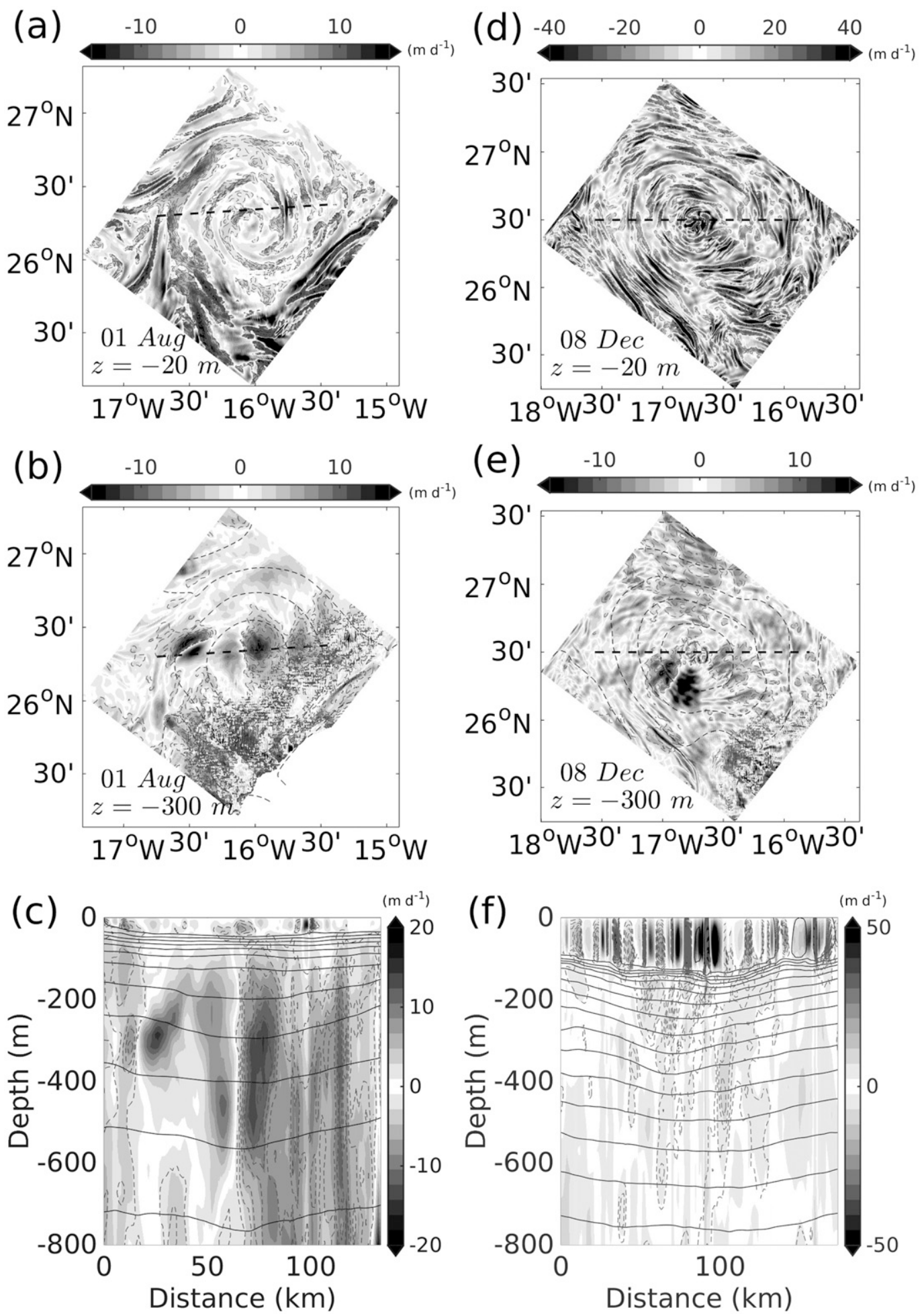

FIG. 7. Horizontal snapshots of vertical velocity $w_{\text {ROMS }}\left(\mathrm{m} \mathrm{day}^{-1}\right)$ for the $\mathrm{A} 2$ vortex for two dates and depths: before the transition time ( $1 \mathrm{Aug}$ ) at (a) $z=-20 \mathrm{~m}$ and (b) $z=-300 \mathrm{~m}$, with (c) the surface vertical section denoted as the dashed line in the horizontal snapshots, and (d)-(f) as in (a)-(c), but for a date after the transition time $(8 \mathrm{Dec})$. Dashed light gray contours indicate negative values of vertical velocity. Superimposed dashed contours in (b) and (e) denote the free surface elevation $\eta(\mathrm{m})$. Gray contours in (c) and (f) are isopycnals plotted every $0.25 \mathrm{~kg} \mathrm{~m}^{-3}$. 

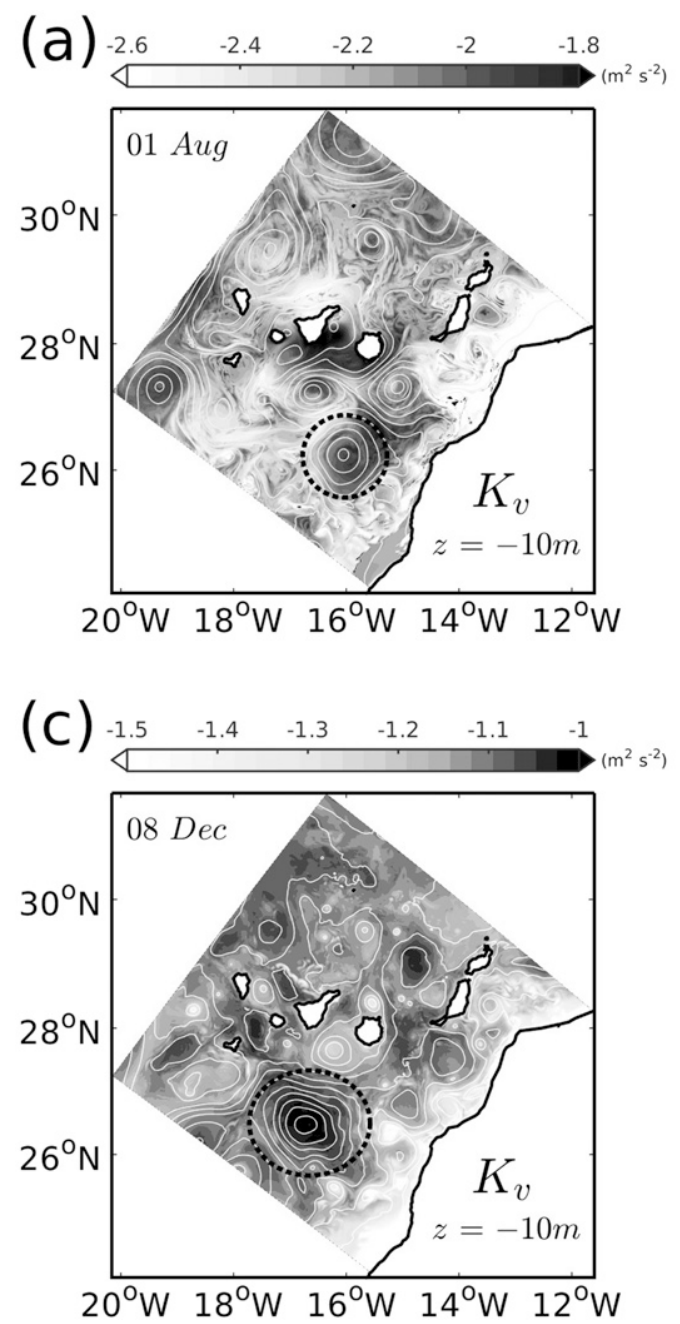
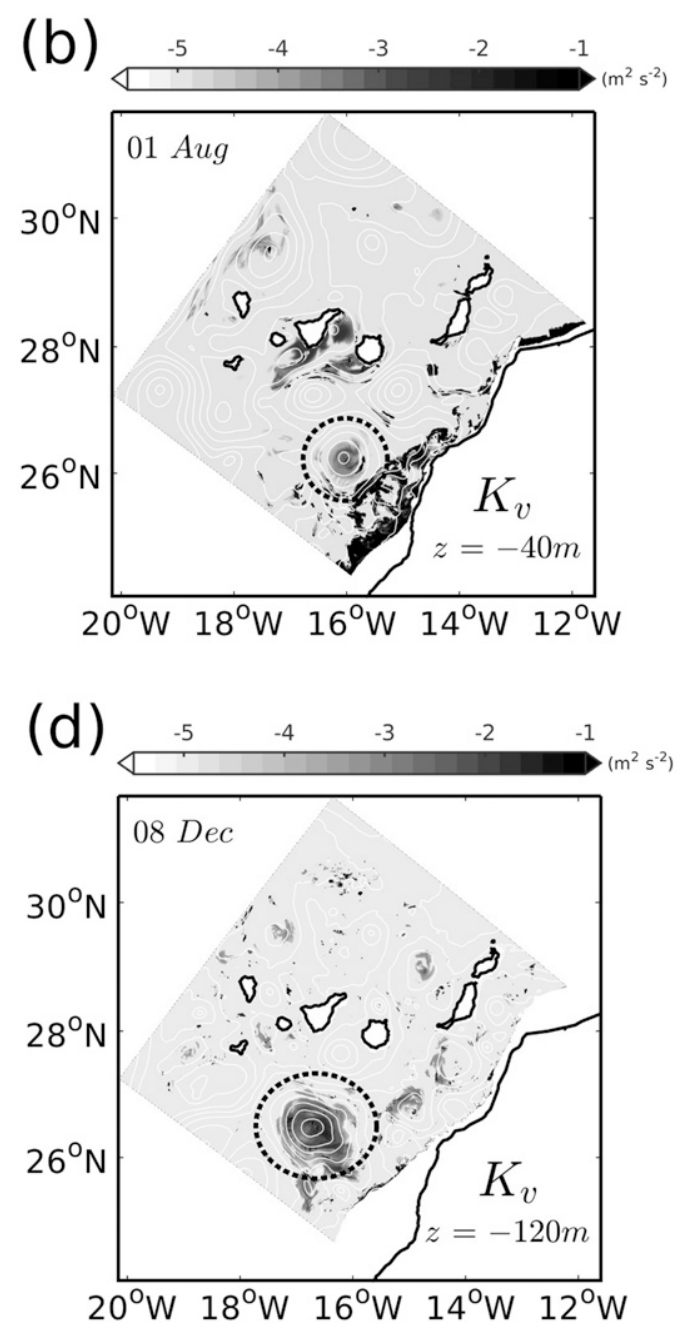

FIG. 8. Snapshots of the diapycnal diffusivity coefficient $K_{v}$ in logarithmic scale, for two dates and depths: before the transition time ( $1 \mathrm{Aug}$ ) at (a) $z=-10 \mathrm{~m}$ in the mixed layer and (b) $z=-40 \mathrm{~m}$ below the mixed layer, and after the transition time ( $8 \mathrm{Dec}$ ) at (c) $z=-10 \mathrm{~m}$ and (d) $z=-120 \mathrm{~m}$ below the mixed layer, covering the entire numeric domain. White contours show the free surface elevation $\eta(\mathrm{m})$. Black dashed circles denote the eddy A2 analyzed in this study. Note changes in the color bar.

indirectly control the magnitude and distribution of $w_{\text {ROMS }}$ by influencing the $w_{\text {Ageo }}$ contribution term (see Fig. 11a). Vertical mixing acts as a source of ageostrophic velocity breaking the thermal-wind balance by smoothing of the vertical velocity profiles (Ponte et al. 2013). Consequently, vertical mixing directly drives ageostrophic motions that increase the ageostrophic term, and hence the total vertical velocity in the mixed layer (Fig. 11a). Moreover, the spiral vertical velocity pattern for $w_{\text {Vmix }}$ resembles the near-surface $w_{\text {ROMS }}$ field (Figs. 3a,f).

Subsurface maxima in $w_{\text {Ageo }}$ and $w_{\text {Vmix }}$ at around $30-\mathrm{m}$ depth in Fig. 11a are suggestive of a TTW balance, which could also explain the secondary circulation in the upper layers of the eddy. TTW is obtained by considering the vertical mixing of momentum in the thermal-wind balance equations. Gula et al. (2014) show that a TTW balance could adequately describe the vertical velocity patterns where the vertical eddy viscosity is large in the upper boundary layer. However, TTW is often found in submesoscale strain-induced fronts and filaments that occur at the eddy peripheries. The results shown here point out that the enhancement of the ageostrophic term due to vertical mixing occurs within the full mixed layer of the eddy, and is not restricted only to the regions between the eddies and their boundaries.

The base of the mixed layer appears to impose a barrier to the extension of the vertical velocity bands which drastically fade below $h_{\text {ubl }}$ (Figs. 7c,f and 11b,c). This is due to the absence of sources of turbulent kinetic 

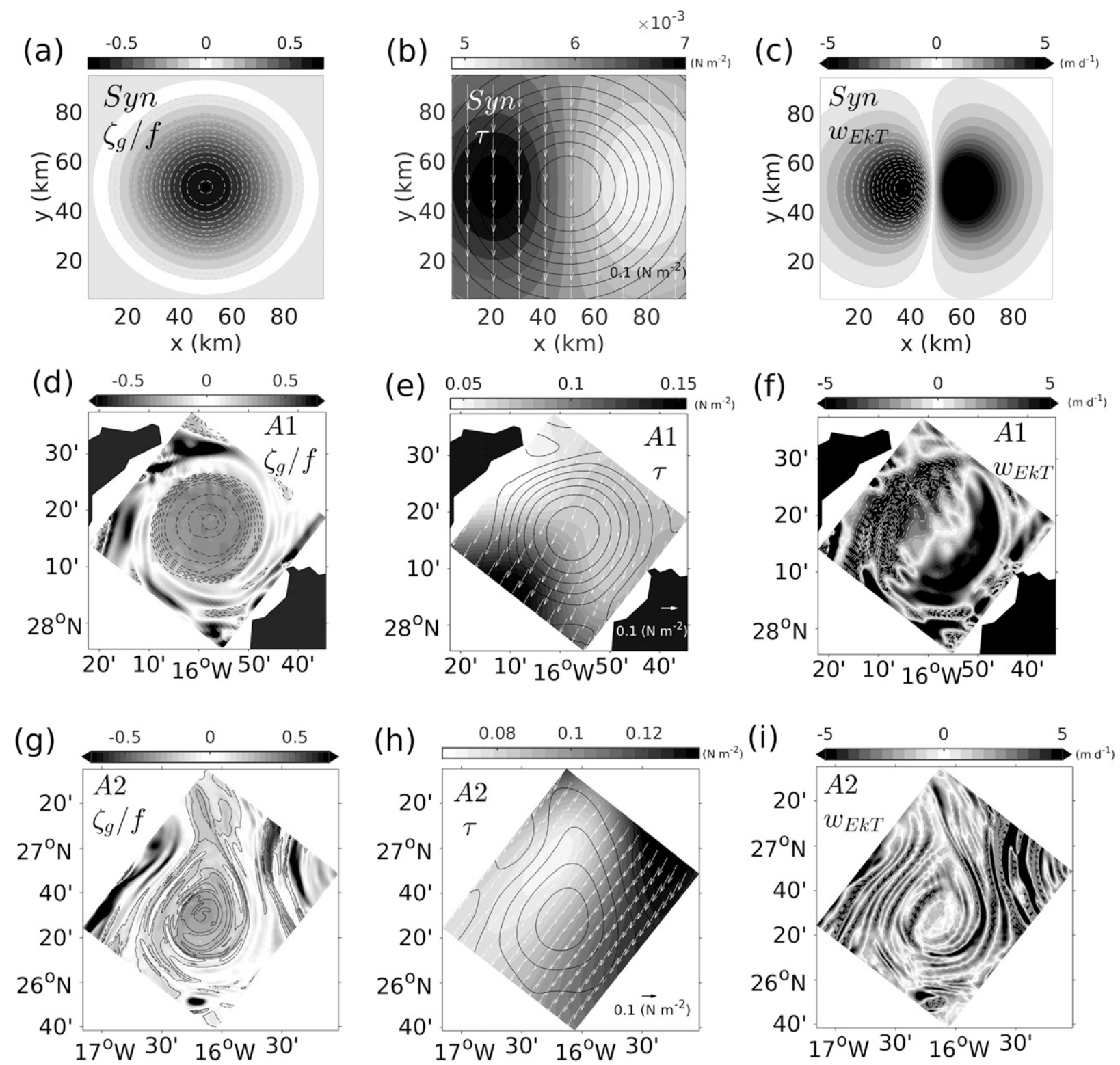

FIG. 9. For three different vortices, (top) a synthetic Gaussian vortex, (middle) the A1 vortex, and (bottom) the A2 vortex, respectively, (a),(d),(g) the geostrophic vertical component of the relative vorticity normalized by the Coriolis frequency ( $/ / f)$, and (b),(e),(h) the wind stress vectors $\boldsymbol{\tau}\left(\mathrm{N} \mathrm{m}^{-2}\right)$, with the wind stress magnitude in grayscale. Black contours are $\eta(\mathrm{m})$ in $(\mathrm{b})-(\mathrm{h})$. A uniform wind field is used for (a); (c),(f),(i) the total Ekman pumping $w_{\mathrm{EkT}}\left(\mathrm{m} \mathrm{day}^{-1}\right)$ by computing Eq. (2). Superimposed dashed gray contours denote negative values of Ro and vertical velocity. Both vortices A1 and A2 are highlighted in Fig. 1a.

energy at the base of the mixed layer. These sources may be vertical shear driven by direct wind erosion and nearinertial vertical shear confined within the mixed layer. Because near-inertial waves inside anticyclonic eddies do not remain in the mixed layer for a long time, there are no sources of near-inertial vertical shear available for mixing and entrainment (Jaimes and Shay 2010).

There is a transition time since the end of August when the regime of the contribution terms for the vertical velocity changes. Near the surface the vertical mixing increases in magnitude with the vertical velocity. Hence, it is expected that an enhancement of $w_{\text {Vmix }}$ reinforces the magnitude of $w_{\text {Ageo }}$ and, as a consequence, there is less cancellation with $w_{\text {Str }}$. This results also in larger $w_{\text {ROMS }}$ in the upper layer (Fig. 11a) as a consequence of the thermal-wind restoring balance. Notice that vertical mixing is intensified as the mixed layer depth deepens toward autumn/winter (Figs. 2a and 8). 
(a) 20
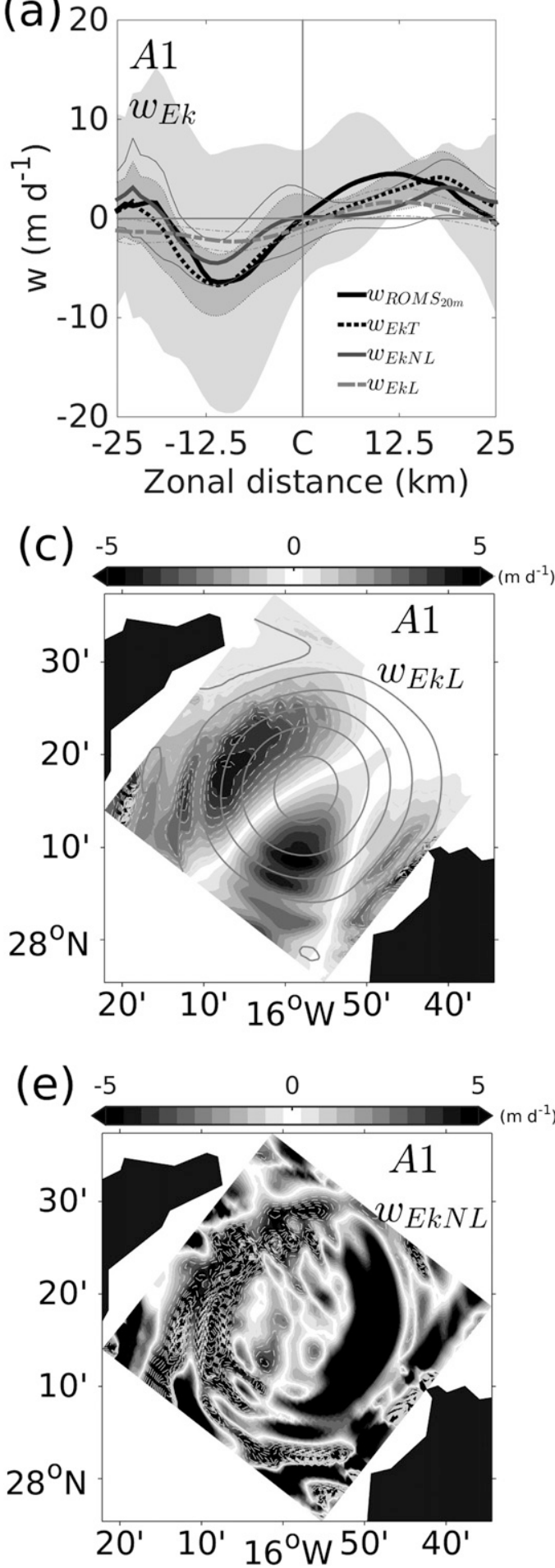

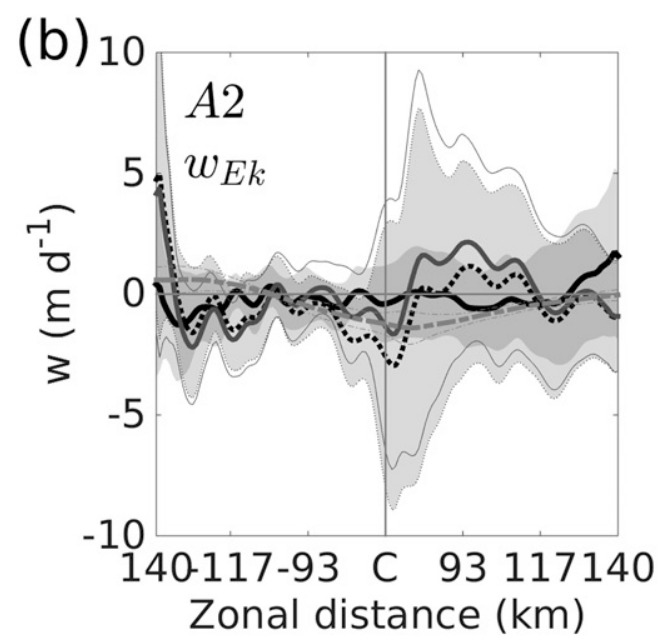

(d) \begin{tabular}{lll}
-5 & 0 & 5 \\
\hline
\end{tabular}
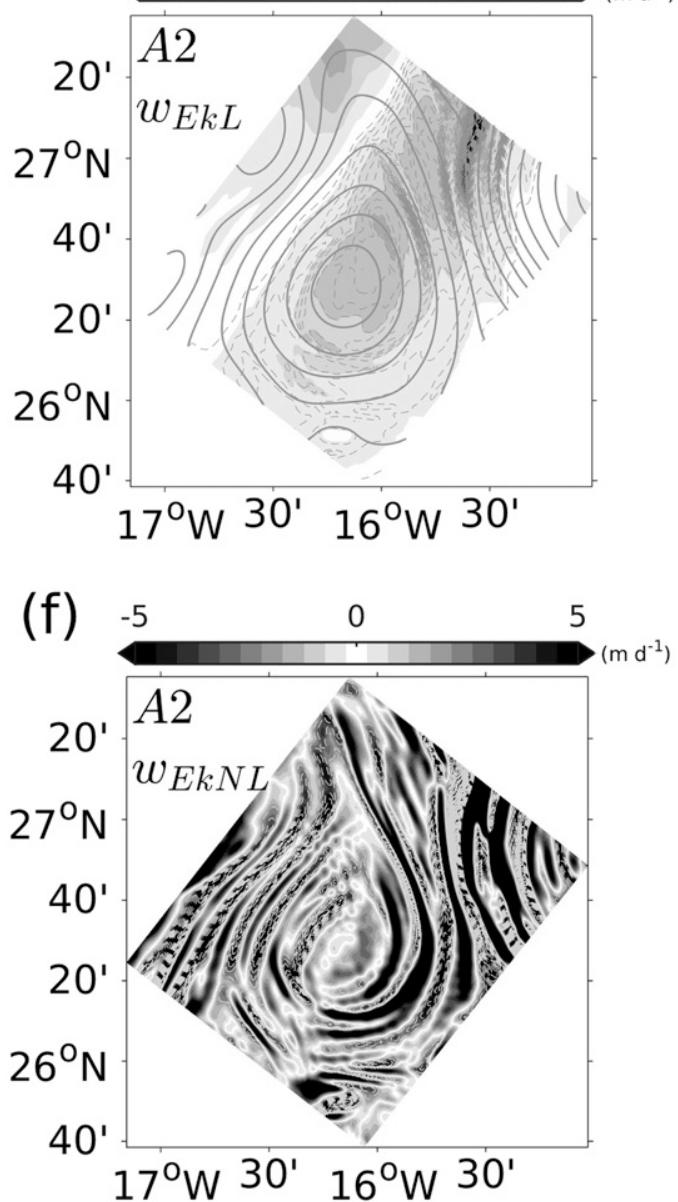

FIG. 10. Latitudinal averages of the $w_{\text {ROMS }}$ at the Ekman layer depth, total Ekman pumping $w_{\mathrm{EkT}}$, nonlinear Ekman pumping $w_{\text {EkNL }}$, and the linear Ekman pumping $w_{\text {EkL }}\left(\right.$ all in $\mathrm{m} \mathrm{day}{ }^{-1}$ ) for (a) the A1 eddy and (b) the A2 eddy. Shaded areas denote the standard deviation error. Vertical dashed lines show vortex centers. The distance from the eddy center $(\mathrm{km})$ is shown at the bottom. Instantaneous snapshots $(29 \mathrm{Aug})$ of (c) $w_{\mathrm{EkL}}$ and (e) $w_{\mathrm{EkNL}}\left(\mathrm{m} \mathrm{day}^{-1}\right)$ are depicted for the A1 eddy. (d),(f) As in (c) and (e), but for the A2 eddy. Gray contours in (c) and (d) are the free surface elevation $\eta(\mathrm{m})$. Superimposed dashed contours denote negative values of vertical velocity. 


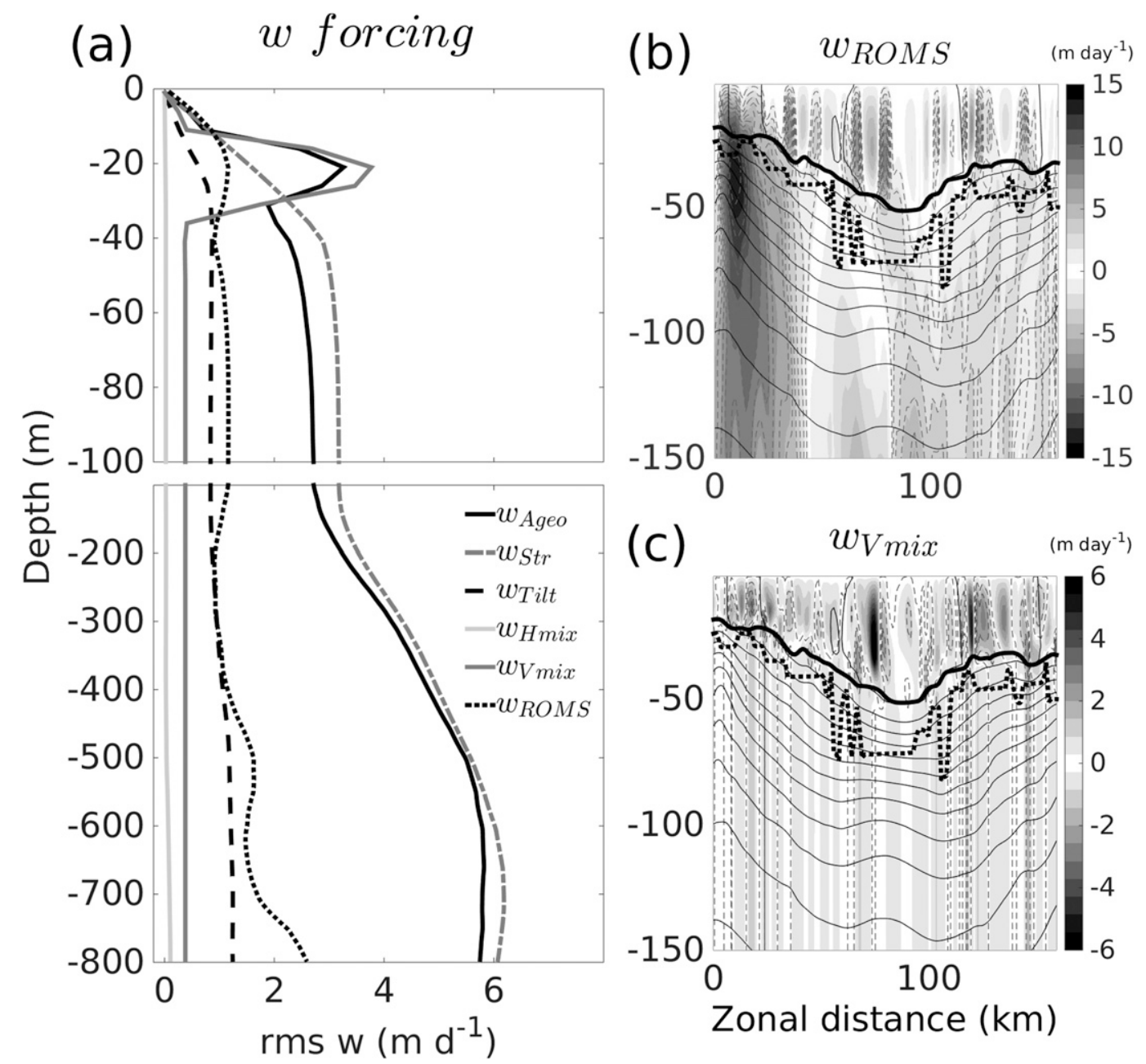

FIG. 11. (a) Rms of each contribution term to the vertical velocity $w_{\text {ROMS }}$ for the A2 eddy. Snapshots of zonal vertical sections at upper layers of the $\mathrm{A} 2$ eddy with (b) $w_{\text {ROMS }}\left(\mathrm{m} \mathrm{day}^{-1}\right.$ ), and (c) vertical mixing contribution term $w_{\text {Vmix }}\left(\mathrm{m} \mathrm{day}^{-1}\right)$. Horizontal continuous and dashed black lines denote the upper boundary layer $h_{\mathrm{ubl}}(\mathrm{m})$ and the surface maximum gradient of the buoyancy frequency squared $N^{2}\left(\mathrm{~s}^{-2}\right)$, respectively. Dashed gray contours denote negative values of vertical velocity. Gray continuous contours are isopycnals plotted every $0.15 \mathrm{~kg} \mathrm{~m}^{-3}$. The zonal section is delimited by the black line shown in Fig. 1.

The forcing time variations in the mixed layer are consistent with the seasonality found in submesoscale regimes reported in recent modeling studies (Mensa et al. 2013; Brannigan et al. 2015) and observational studies (Callies et al. 2015; Buckingham et al. 2016), where the variance of Ro numbers and mixing are enhanced in winter times.

\section{b. Influence of Ekman pumping on the vertical velocity field}

The total Ekman pumping is mainly driven by nonlinear Ekman term, in agreement with the general magnitude/ pattern of $w_{\text {ROMS }}$ in the mixed layer (see Figs. 3a and 9i). The dominance of nonlinear Ekman pumping is related to ageostrophic dynamics for large Ro, causing the Ekman transport divergence to vary proportionally to the inverse absolute relative vorticity $(f+\zeta)$ rather than only planetary vorticity (Niiler 1969; Stern 1965; Thomas and Rhines 2002). This term includes the tilting of vertical relative vorticity, which allows for vertical velocities even with horizontally uniform wind stress (Stern 1965). The other important effect is that for high Ro flow structures the horizontal gradients of vertical vorticity are enhanced, probably increasing the advection of vertical vorticity by Ekman transport. Both effects can change the magnitude of nonlinear Ekman pumping. The averaged linear Ekman pumping field shows an inverted normal distribution rather than a Gaussian distribution as theorized by McGillicuddy et al. (2008), likely due to a nonuniform southwestward wind field. The results indicate that although vertical velocity can be related with nonlinear Ekman pumping at the 

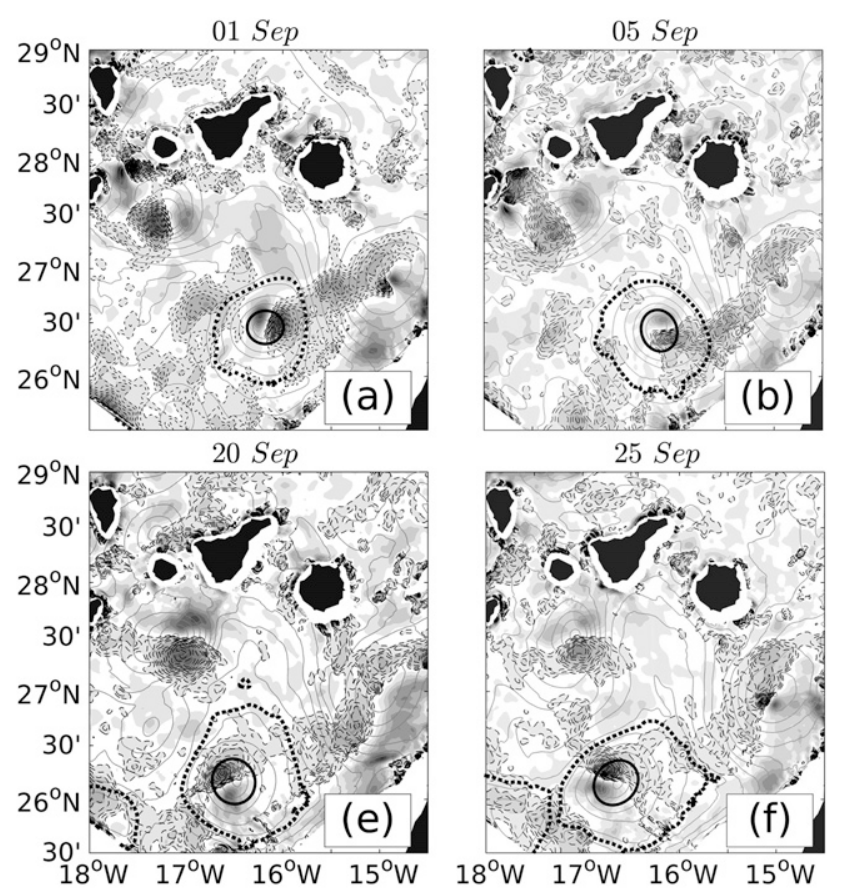

$25 \mathrm{Sep}$
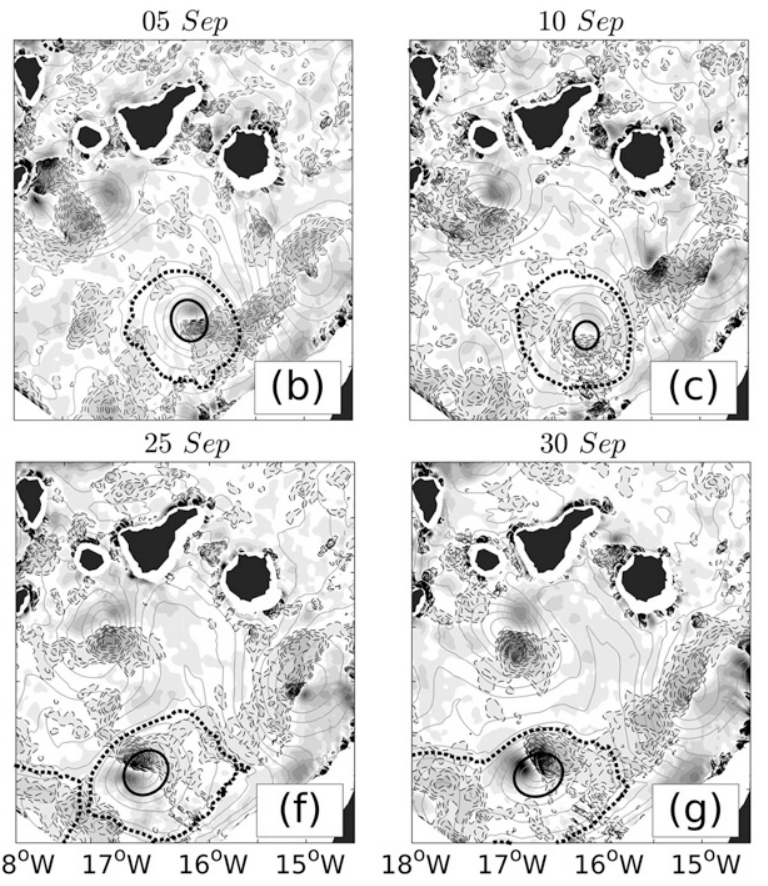

$30 \mathrm{Sep}$

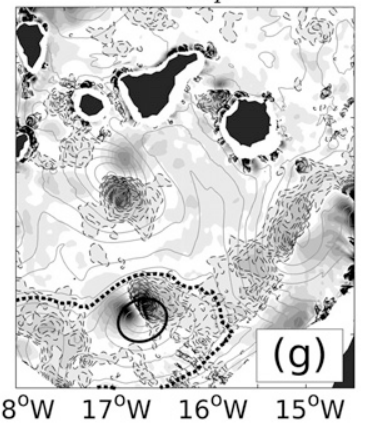

$18^{\circ} \mathrm{W} \quad 17^{\circ} \mathrm{W} \quad 16^{\circ} \mathrm{W} \quad 15^{\circ} \mathrm{W}$

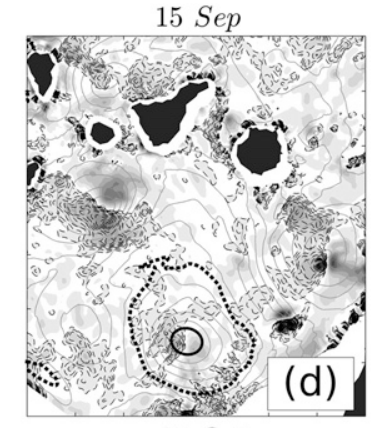

05 Oct

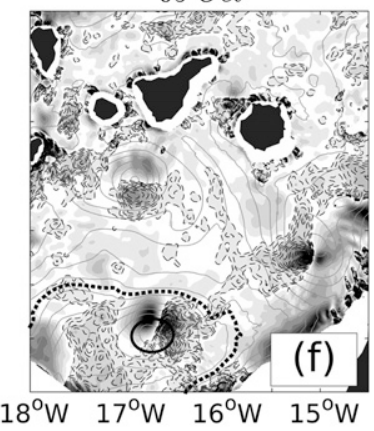

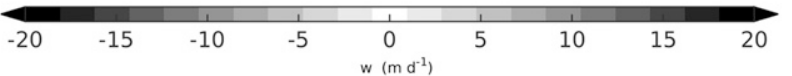

FIG. 12. Temporal evolution of the vertical velocity dipolar structure for various eddies south of the Canary Islands. Snapshots correspond to horizontal maps of vertical velocity $w_{\mathrm{ROMS}}\left(\mathrm{m} \mathrm{day}^{-1}\right)$, at $z=-300 \mathrm{~m}$, plotted every 5 days from 1 Sep. Potential density anomaly $\sigma_{\theta}\left(\mathrm{kg} \mathrm{m}^{-3}\right)$, at $300-\mathrm{m}$ depth is plotted as continuous gray contours with an interval of $0.02 \mathrm{~kg} \mathrm{~m}^{-3}$. The eddy-core edge fitted to an ellipse is delimited in black. The dashed black contour is the isopycnal $26.87 \mathrm{~kg} \mathrm{~m}^{-3}$. Superimposed dashed gray contours are negative values of vertical velocity.

mixed layer, this process is not able to explain the dipole pattern of the vertical velocity at the thermocline layers.

It is worth considering the validity of Stern (1965)'s formulation of the Ekman pumping for anticyclonic curved flows with Ro numbers approaching unity, such as the case here. McWilliams (2017) shows that important nonlinearities arise in the surface boundary layer current dynamics if Ro is large. Wenegrat and Thomas (2017) recently extended Stern's (1965) formulation to consider the high-order effects in flows with curvature. They pointed out that including higherorder terms in Ro number on the Ekman pumping velocity leads to an improvement in accuracy. The authors show a reduction (increase) of the magnitude of the Ekman pumping in a circular submesoscale anticyclonic (cyclonic) vortex, which is a consequence of the pumping in Eq. (2) (Stern 1965). Testing the accuracy of the Ekman pumping in the numerical anticyclone is outside of the scope of this study, although Wenegrat and Thomas's (2017) formulation should be considered in future work as it would increase the accuracy of the wind-forced vertical velocity in simulated mesoscale eddies.

\section{c. Subsurface vertical velocity dipolar structure}

The dipolar cell of vertical velocity is mainly forced by the combined effects of stretching and advection of vorticity. The dipolar structure of vertical velocity and the dominance of the ageostrophic forcing terms are also shown by diagnosing the vertical velocity from Eq. (8) from the terms $w_{\mathrm{QA}}$ and $w_{\mathrm{PSE}}$. These terms are analogous to the advection of vorticity and instantaneous changes in the vertical velocity given in Eq. (1). Despite using different approaches, both analyses reveal that ageostrophic forcing and advection of vorticity are relevant for inference of vertical velocity dipoles within mesoscale eddies.

The dipoles are ubiquitous in all the simulated mesoscale eddies (both cyclones and anticyclones), varying in intensity over time (Fig. 12). From wavelet analysis of numerical Lagrangian trajectories (not shown), a fluid particle takes typically about 8 days to do one revolution along the potential vorticity (PV) surfaces of the anticyclonic eddy. This rotation rate matches rather well with those observed by Sangrà et al. $(2005,2007)$ from drifters deployed inside Canary Island eddies. By contrast, the vertical velocity dipolar structures revolve 

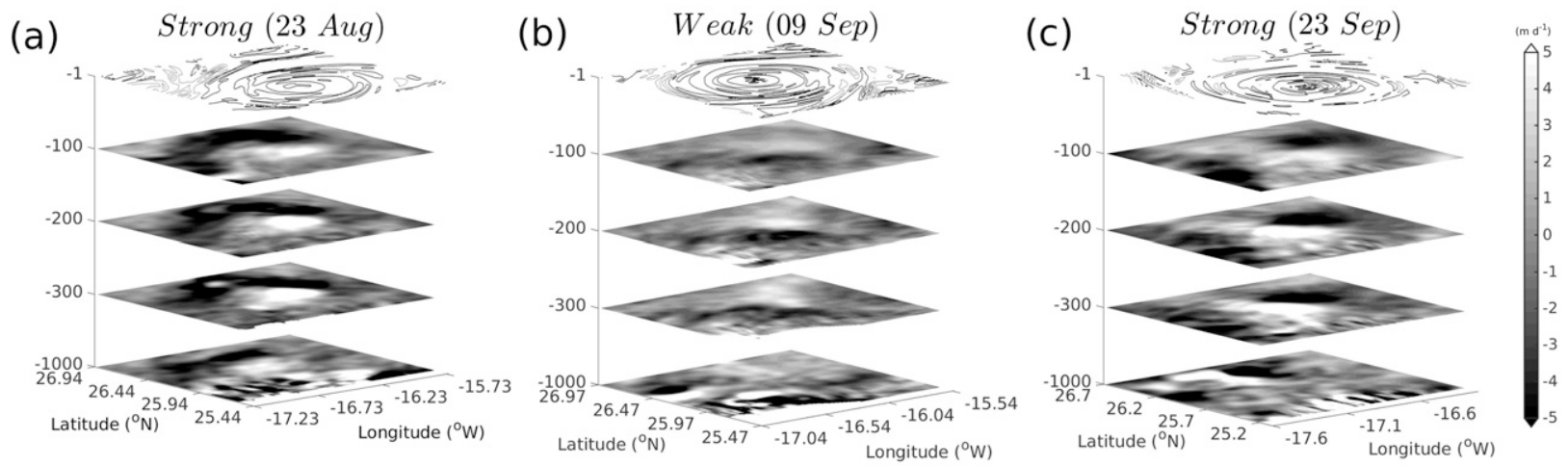

FIG. 13. Horizontal maps of vertical velocity $w_{\text {ROMS }}\left(\mathrm{m} \mathrm{day}^{-1}\right)$, for the A2 eddy at four different depths: $-100,-200$, -300 , and $-1000 \mathrm{~m}$. To show variations in the magnitude of the vertical velocity dipolar structure, three dates are taken into account: (a) 23 Aug, (b) 9 Sep, and (c) 23 Sep. Contours of relative vorticity $\zeta\left(\mathrm{s}^{-1}\right)$ are represented in gray (black) for $\zeta>0(\zeta<0)$

anticyclonically with a slower speed, taking $\sim 30$ days to complete a cycle as shown in the time evolution of vertical velocity in the anticyclone A2 (Fig. 12).

The vertical velocity dipoles also experience changes in their vertical velocity magnitude with time. Figure 12 shows that it weakens on 10 September (Fig. 12c) but is then reinforced 10 days later (Fig. 12e) and maintains its intensity for two weeks (Figs. 12e-h). Similarly, the weakening/strengthening of the vertical velocity dipole is shown in Fig. 13. Weaker stages of vertical velocity dipoles should not be attributed to a shallow vertical extension of the dipole in the water column since its vertical extension appears to be constant along the eddy lifespan. This is shown in Fig. 13, where the vertical velocity dipole between $z=100-300 \mathrm{~m}$ weakens from 23 August (Fig. 13a) to 9 September (Fig. 13b). After $\approx 14$ days, the vertical velocity dipole strengthens again on 23 September (Fig. 13c) and maintains this intensity for 30 days. Below the eddy $(z=-1000 \mathrm{~m})$, vertical velocity dipoles are also ubiquitous but decoupled with the ones associated to the surface intensified anticyclonic eddy A2 (Fig. 13).

Another issue is whether the intensity of the dipoles can be related to the vortex axisymmetric state. In a perfect ellipsoid of PV, potential density $\sigma_{\theta}$ and PV contours are not exactly parallel at the vertexes of the ellipse. As a consequence, the particles that approach a vertex, and move along PV contours faster than the phase speed of the eddy, must climb $(w>0)$ or descend $(w<0)$ the isopycnal surfaces to conserve PV (Viúdez and Dritschel 2003). Regions of crossing $\sigma_{\theta}$ and PV contours also can be interpreted as regions of enhanced dynamical imbalance and hence vertical circulation. To quantify axisymmetrization, the eddy-core edge is defined as the isopycnal along which the average of the horizontal velocity magnitude is maximum (Chaigneau et al. 2011). Next, the isopycnal contour can be fitted to an ellipse (black contours in Fig. 12) to obtain the eccentricity $\varepsilon$ that represents changes in the axisymmetrization period of the eddy. Qualitative visual examination of $w_{\text {ROMS }}$ and the fitted ellipses in Fig. 12, suggests that the eddy's axisymmetry is correlated with a weak vertical velocity (Figs. 12c,d for vortex A2). Results indicate that $\varepsilon=0.44$ for weak vertical velocity $\left(\mathrm{rms}=2.64 \mathrm{~m} \mathrm{day}^{-1}\right)$ (Fig. 12c),$\varepsilon=0.66$ for moderate vertical velocity $\left(\mathrm{rms}=5.48 \mathrm{~m} \mathrm{day}^{-1}\right)$ (Fig. 12f), and $\varepsilon=0.76$ for strong dipolar structures of vertical velocity $\left(\mathrm{rms}=6.60 \mathrm{~m} \mathrm{day}^{-1}\right)$ (Fig. 12h). The same is found for results of Figs. 13a-c, with $\varepsilon$ of $0.71,0.47$, and 0.60 corresponding to rms of vertical velocities of 5.20, 2.93, and $5.14 \mathrm{~m} \mathrm{day}^{-1}$, respectively. This suggests that vertical velocities in the pycnocline may be strengthened when the eddy loses its axisymmetrization to become a less circular eddy. Notice that for single signed PV elliptical balls the distribution of vertical velocity is quadrupolar (Viúdez and Dritschel 2003) and contrasts with the dipolar distribution found here. Differences may be because anticyclone A2 1) is not a single signed PV ball but an isolated eddy with positive vorticity on the outer edge, 2) is not a symmetric ellipsoid but rather a deformed circular eddy, and 3 ) is forced by climatological winds.

In general, the ageostrophic and stretching terms are the dominant forcing of the $w_{\text {ROMS }}$ field at the thermocline and remain almost constant during the transition time (Fig. 6b). However, the $w_{\text {Tilt }}$ and $w_{\text {Vmix }}$ forcing terms increase resulting in larger small-scale vertical velocity and inducing more patchy, but enhanced, dipolar vertical velocity. Thereby, it seems that both forcings, along with changes of the vortex axisymmetrization, can interplay to maintain or diffuse the vertical velocity pattern within the eddy. Recently, Viúdez (2017), in an idealized numerical study of marginal isolated vortices, suggests that vertical velocity dipolar patterns are related with the axial precession (likely by 
vortex tilting) of a spheroidal vortex. This cause the inclination of the isopycnals that ultimately leads to the upward and downward motion of fluid particles. How these processes impact on the vertical velocity dipole dynamics within eddies is not a straightforward issue and must be analyzed in detail in future studies.

\section{Concluding remarks}

The main results of this study indicate that the vertical velocity inside an anticyclonic eddy in the CEC is strongly linked to ageostrophic effects. Nonlinear Ekman pumping (Stern 1965) contributes significantly to the filamentary structures of vertical velocity within the mixed layer of the simulated anticyclonic eddies, although it overestimates the magnitude (see Wenegrat and Thomas 2017).

In terms of vertical velocity structure, two different vertical velocity patterns are found inside the simulated eddies. In the mixed layer, vertical velocity exhibits a wavelike pattern with alternating filaments of downwelling/upwelling cells around the eddy core. This pattern is triggered by vertical mixing at moderately high Ro and variable $K_{v}$, which indirectly controls vertical velocity by enhancing the ageostrophic contribution term and breaking thermal-wind balance. The temporal evolution of the vertical velocity contribution terms shows that vertical mixing can modulate the magnitude of the ageostrophic term. Therefore, increases of nearsurface mixing induce enhancements of vertical velocity in agreement with the seasonality of submesoscale structures.

In the thermocline, a recurrent dipole-like structure of vertical velocity dominates the core of the analyzed eddy. The dipolar structure rotates anticyclonically around the eddy center and has a mesoscale smoothed structure (contrary to the small-scale structure of the vertical velocity in the mixed layer). A vertical velocity dipole within a CEC anticyclonic eddy was recently reported from in situ density and horizontal velocity by Barceló-Llull et al. (2017). The vertical velocity dipolar distribution is mainly induced by the advection of vorticity through a combined effect between the ageostrophic and stretching terms. Results suggest that the dipole becomes more patchy and diffuse, though intensifying its magnitude, as the tilting and vertical mixing terms increase during autumn and winter times. This can also be related with cycles in the eddy axisymmetrization as the dipole of vertical velocity is reinforced with elliptical phases of the eddy.

The vertical velocity dipole is also obtained when a generalized omega equation is solved for ROMS's density and horizontal velocity. The results show that the use of the generalized omega equation leads to consistent estimates of the magnitude of the vertical velocity field below the mixed layer inside the mesoscale eddy. Moreover, analysis of the omega equation forcing terms shows that the divergence of the total $\mathbf{Q}$ vector dominates the vertical velocity field. The straining deformation is mainly induced by the ageostrophic horizontal field. The term $w_{\mathrm{QA}}$ dominates the upper $200 \mathrm{~m}$ of the water column, and $w_{\text {PSE }}$ controls the $w_{\text {OMEGA }}$ field below $200 \mathrm{~m}$. This indicates that the ageostrophic terms are the main forcings on the distribution of the vertical velocity within the simulated mesoscale eddy in agreement with the analysis of vertical velocity through integrating the divergence equation vertically.

In conclusion, we suggest that the vertical velocity dipolar structures within mesoscale eddies are due to the sum of the ageostrophic and stretching terms, which are a similar forcing term to the divergence of the ageostrophic $\mathbf{Q}$ vector plus the pseudovorticity forcing term or the ageostrophic advection of relative vorticity.

Acknowledgments. This work has been supported by the FLUXES (CTM2015-69392-C3-3-R) project funded by the Spanish National Research Program and the European Regional Development Fund (MINECOFEDER). Thanks to the essential support of Pablo Sangrà, from now on always in our memories. Sheila Estrada-Allis is supported by a post-doctoral contract from the National Council of Science and Technology of Mexico-Mexican Ministry of Energy-Hydrocarbon Trust, project 201441. This is a contribution of the Gulf of Mexico Research Consortium (CIGoM). The modeling data necessary to reproduce this study were provided by Dr. Evan Mason (evanmason@gmail.com). Evan Mason is supported by the Copernicus Marine Environment Monitoring Service (CMEMS) MedSUB project; CMEMS is implemented by Mercator Ocean in the framework of a delegation agreement with the European Union. Bàrbara Barceló-Llull is supported by a post-doctoral contract from the Spanish National Research Program associated to the PRESWOT project (CTM2016-78607-P). Thanks to Inga Koszalka for useful comments at an earlier stage of the paper.

\section{APPENDIX}

\section{Derivation of Eq. (1)}

We decompose the vertical velocity field similarly as in Koszalka et al. (2009) (their appendix A). The differences in derivation and final expression of Eq. (1) are explained below. 
Horizontal momentum conservation equations under Boussinesq approximation are:

$x$ horizontal momentum

$$
\begin{aligned}
\frac{D u}{D t}-f v= & -\frac{1}{\rho_{o}} \frac{\partial p}{\partial x}+\frac{\partial}{\partial x}\left(A_{H} \frac{\partial u}{\partial x}\right)+\frac{\partial}{\partial y}\left(A_{H} \frac{\partial u}{\partial y}\right) \\
& +\frac{\partial}{\partial z}\left(K_{v} \frac{\partial u}{\partial z}\right), \text { and }
\end{aligned}
$$

$y$ horizontal momentum

$$
\begin{aligned}
\frac{D v}{D t}+f u= & -\frac{1}{\rho_{o}} \frac{\partial p}{\partial y}+\frac{\partial}{\partial x}\left(A_{H} \frac{\partial v}{\partial x}\right)+\frac{\partial}{\partial y}\left(A_{H} \frac{\partial v}{\partial y}\right) \\
& +\frac{\partial}{\partial z}\left(K_{v} \frac{\partial v}{\partial z}\right),
\end{aligned}
$$

with $D / D t=\partial / \partial t+u \partial / \partial x+v \partial / \partial y+w \partial / \partial z$ as the material derivative; $A_{H}$ is the horizontal mixing coefficient, $K_{v}$ is the vertical mixing coefficient computing using the KPP (Large et al. 1994), $\mathbf{U}=(u, v, w)$ is the three-dimensional velocity field, $f$ is the Coriolis parameter, $p$ is the pressure, and $\rho_{o}$ is a constant reference density $\left(1026 \mathrm{~kg}^{-1} \mathrm{~m}^{-3}\right)$.

The curl of Eqs. (A1) and (A2) through crossdifferentiation by $\partial / \partial y$ and $-\partial / \partial x$ yields

$$
\begin{aligned}
-\frac{\partial v}{\partial y}\left(f-\frac{\partial u}{\partial y}\right)= & \frac{D}{D t}\left(-\frac{\partial u}{\partial y}\right)+\frac{\partial u}{\partial x}\left(-\frac{\partial u}{\partial y}\right)-\frac{1}{\rho_{o}} \frac{\partial}{\partial y} \frac{\partial p}{\partial x} \\
& -\left(\frac{\partial w}{\partial y} \frac{\partial u}{\partial y}\right)-A_{H}\left(\frac{\partial^{4}}{\partial x^{4}} \frac{\partial^{4}}{\partial y^{4}}\right)\left(-\frac{\partial u}{\partial y}\right) \\
& +\frac{\partial}{\partial y} \frac{\partial}{\partial z}\left(K_{v} \frac{\partial u}{\partial z}\right), \text { and } \\
-\frac{\partial u}{\partial x}\left(f+\frac{\partial v}{\partial x}\right)= & \frac{D}{D t}\left(-\frac{\partial v}{\partial x}\right)+\frac{\partial v}{\partial y}\left(\frac{\partial v}{\partial x}\right)+\frac{1}{\rho_{o}} \frac{\partial}{\partial x} \frac{\partial p}{\partial y} \\
& +\left(\frac{\partial w}{\partial x} \frac{\partial v}{\partial z}\right)-A_{H}\left(\frac{\partial^{4}}{\partial x^{4}} \frac{\partial^{4}}{\partial y^{4}}\right)\left(\frac{\partial v}{\partial x}\right) \\
& -\frac{\partial}{\partial x} \frac{\partial}{\partial z}\left(K_{v} \frac{\partial v}{\partial z}\right) .
\end{aligned}
$$

After a little algebra, Eqs. (A3) and (A4) can be written as

$$
\begin{aligned}
-\frac{\partial v}{\partial x}\left(f+\zeta_{2}\right)= & {\left[\frac{D \zeta_{2}}{D t}+\frac{\partial u}{\partial x} \zeta_{2}-\frac{1}{\rho_{o}} \frac{\partial}{\partial y} \frac{\partial p}{\partial x}\right.} \\
& -\frac{\partial w}{\partial y} \frac{\partial u}{\partial z}-A_{H}\left(\frac{\partial^{4}}{\partial x^{4}} \frac{\partial^{4}}{\partial y^{4}}\right) \zeta_{2} \\
& \left.+\frac{\partial}{\partial y} \frac{\partial}{\partial z}\left(K_{v} \frac{\partial u}{\partial z}\right)\right], \text { and }
\end{aligned}
$$

$$
\begin{aligned}
-\frac{\partial u}{\partial y}\left(f+\zeta_{1}\right)= & {\left[\frac{D \zeta_{1}}{D t}+\frac{\partial v}{\partial y} \zeta_{1}+\frac{1}{\rho_{o}} \frac{\partial}{\partial x} \frac{\partial p}{\partial y}\right.} \\
& +\frac{\partial w}{\partial x} \frac{\partial v}{\partial z}-A_{H}\left(\frac{\partial^{4}}{\partial x^{4}} \frac{\partial^{4}}{\partial y^{4}}\right) \zeta_{1} \\
& \left.-\frac{\partial}{\partial x} \frac{\partial}{\partial z}\left(K_{v} \frac{\partial v}{\partial z}\right)\right],
\end{aligned}
$$

where $\zeta_{1}=\partial v / \partial x$ and $\zeta_{2}=-\partial u / \partial y$, and $\zeta=\zeta_{1}+\zeta_{2}$ is the vertical component of the relative vorticity. Notice that the pressure terms are canceled when subtracting.

To obtain Eq. (1), Eq. (A5) is divided by $\left(f+\zeta_{2}\right)$ and Eq. (A6) by $\left(f+\zeta_{1}\right)$ and summed to give $\partial w / \partial z=$ $-\partial u / \partial x-\partial v / \partial y$. Vertical integration of the latter expression (i.e., the divergence of the flow) is used to diagnose the vertical velocity in a hydrostatic Boussinesq primitive equation framework.

In contrast to Koszalka et al. (2009), the wind effect on the vertical velocity is not included explicitly in Eq. (1) [cf. Eqs. (A1) and (A2) in this appendix with the ones of appendix A in Koszalka et al. (2009)] and it is analyzed separately, in an Ekman layer dynamics sense. Moreover, the stretching term is joined to the ageostrophic terms in the analysis, since their tendency is to cancel each other (Koszalka et al. 2009).

\section{REFERENCES}

Arístegui, J., P. Sangrà, S. Hernádez-León, M. Cantón, A. Hernández-Guerra, and J. L. Kerling, 1994: Island-induced eddies in the Canary Island. Deep-Sea Res. I, 41, 1509-1525, https://doi.org/10.1016/0967-0637(94)90058-2.

Barceló-Llull, B., E. Pallàs-Sanz, P. Sangrà, A. Martínez-Marrero, S. Estrada-Allis, and J. Arístegui, 2017: Ageostrophic secondary circulation in a subtropical mode water eddy. J. Phys. Oceanogr., 47, 1107-1123, https://doi.org/10.1175/JPO-D-16-0235.1.

Brannigan, L., D. Marshall, A. Naveira-Garabato, and A. Nurser, 2015: The seasonal cycle of submesoscale flows. Ocean Modell., 92, 69-84, https://doi.org/10.1016/j.ocemod.2015.05.002.

Buckingham, C., and Coauthors, 2016: Seasonality of submesoscale flows in the ocean surface boundary layer. Geophys. Res. Lett., 43, 2118-2126, https://doi.org/10.1002/2016GL068009.

Calil, P. H. R., and K. J. Richards, 2010: Transient upwelling hot spots in the oligotrophic North Pacific. J. Geophys. Res., 115, C02003, https://doi.org/10.1029/2009JC005360.

Callies, J., R. Ferrari, J. Klymak, and J. Gula, 2015: Seasonality in submesoscale turbulence. Nat. Commun., 6, 6862, https:// doi.org/10.1038/ncomms7862.

Capet, X., J. C. McWilliams, M. J. Molemaker, and A. F. Shchepetkin, 2008: Mesoscale to submesoscale transition in the California Current System. Part II: Frontal processes. J. Phys. Oceanogr., 38, 44-64, https://doi.org/10.1175/2007JPO3672.1.

Cardona, Y., and A. Bracco, 2012: Enhanced vertical mixing within mesoscale eddies due to high frequency winds in the South China Sea. Ocean Modell., 42, 1-15, https://doi.org/10.1016/ j.ocemod.2011.11.004.

Chaigneau, A., M. Le Texier, G. Eldin, C. Grados, and O. Pizarro, 2011: Vertical structure of mesoscale eddies in the eastern 
South Pacific Ocean: A composite analysis from altimetry and Argo profiling floats. J. Geophys. Res., 116, C11025, https:// doi.org/10.1029/2011JC007134.

Garrett, C., and J. Loder, 1981: Dynamical aspects of shallow sea fronts. Philos. Trans. Roy. Soc. London, 302B, 563-581, https:// doi.org/10.1098/rsta.1981.0183.

Gaube, P., D. B. Chelton, R. M. Samelson, M. G. Schlax, and L. W. O'Neill, 2015: Satellite observations of mesoscale eddyinduced Ekman pumping. J. Phys. Oceanogr., 45, 104-132, https://doi.org/10.1175/JPO-D-14-0032.1.

Giordani, H., L. Prieur, and G. Caniaux, 2006: Advanced insights into sources of vertical velocity in the ocean. Ocean Dyn., 56, 513-524, https://doi.org/10.1007/s10236-005-0050-1.

Graves, L. P., J. C. McWilliams, and M. T. Montgomery, 2006: Vortex evolution due to straining: A mechanism for dominance of strong, interior anticyclones. Geophys. Astrophys. Fluid Dyn., 100, 151-183, https://doi.org/10.1080/03091920600792041.

Gula, J., M. J. Molemaker, and J. C. McWilliams, 2014: Submesoscale cold filaments in the Gulf Stream. J. Phys. Oceanogr., 44, 2617-2643, https://doi.org/10.1175/JPO-D-14-0029.1.

Haidvogel, D. B., and A. Beckmann, 1999: Numerical Ocean Circulation Modeling. Imperial College Press, $344 \mathrm{pp}$.

Hoskins, B. J., I. Draghici, and H. A. C. Davies, 1978: A new look at the $\omega$-equation. Quart. J. Roy. Meteor. Soc., 104, 31-38, https:// doi.org/10.1002/qj.49710443903.

Jaimes, B., and L. Shay, 2010: Near-inertial wave wake of Hurricanes Katrina and Rita over mesoscale oceanic eddies. J. Phys. Oceanogr., 40, 1320-1337, https://doi.org/10.1175/2010JPO4309.1.

Jiménez, B., P. Sangrà, and E. Mason, 2008: A numerical study of the relative importance of wind and topographic forcing on oceanic eddy shedding by tall, deep water islands. Ocean Modell., 22, 146-157, https://doi.org/10.1016/j.ocemod.2008.02.004.

Johnston, T. M. S., D. L. Rudnick, and E. Pallàs-Sanz, 2011: Elevated mixing at a front. J. Geophys. Res., 116, C11033, https:// doi.org/10.1029/2011JC007192.

Koszalka, I., A. Bracco, J. C. McWilliams, and A. Provenzale, 2009: Dynamics of wind-forced coherent anticyclones in the open ocean. J. Geophys. Res., 114, C08011, https://doi.org/10.1029/ 2009JC005388.

— L. Ceballos, and A. Bracco, 2010: Vertical mixing and coherent anticyclones in the ocean: The role of stratification. Nonlinear Processes Geophys., 17, 37-47, https://doi.org/ 10.5194/npg-17-37-2010.

Lapeyre, G., and P. Klein, 2006: Dynamics of the upper oceanic layers in terms of surface quasigeostrophic theory. $J$. Phys. Oceanogr., 36, 165-176, https://doi.org/10.1175/JPO2840.1.

Large, W. G., J. C. McWilliams, and S. Doney, 1994: Oceanic vertical mixing: A review and a model with a nonlocal boundary layer parameterization. Rev. Geophys., 32, 363-403, https://doi.org/10.1029/94RG01872.

Mahadevan, A., and A. Tandon, 2006: An analysis of mechanisms for submesoscale vertical motion at ocean fronts. Ocean Modell., 14, 241-256, https://doi.org/10.1016/j.ocemod.2006.05.006.

__ L. N. Thomas, and A. Tandon, 2008: Comment on "Eddy/ wind interactions stimulate extraordinary mid-ocean plankton blooms." Science, 320, 448, https://doi.org/10.1126/ science. 1152111.

Martin, A., and K. Richards, 2001: Mechanisms for vertical nutrient transport within a North Atlantic mesoscale eddy. Deep-Sea Res. II, 48, 757-773, https://doi.org/10.1016/S0967-0645(00)00096-5.

Mason, E., 2009: High-resolution modelling of the Canary Basin oceanic circulation. Ph.D. thesis, Universidad de Las Palmas de Gran Canaria, 235 pp.
_ J. Molemaker, A. F. Shchepetkin, F. Colas, J. C. McWilliams, and P. Sangrà, 2010: Procedures for offline grid nesting in regional ocean models. Ocean Modell., 35, 1-15, https://doi.org/ 10.1016/j.ocemod.2010.05.007.

_ , F. Colas, J. Molemaker, A. F. Shchepetkin, C. Troupin, J. McWilliams, and P. Sangrà, 2011: Seasonal variability of the Canary Current: A numerical study. J. Geophys. Res., 116, C06001, https://doi.org/10.1029/2010JC006665.

— _ _ _ and J. L. Pelegrí, 2012: A Lagrangian study tracing water parcel origins in the Canary Upwelling System. Sci. Mar., 76 (Suppl.), 79-94, https://doi.org/10.3989/scimar.03608.18D.

_- A. Pascual, and J. C. McWilliams, 2014: A new sea surface height-based code for oceanic mesoscale eddy tracking. J. Atmos. Oceanic Technol., 31, 1181-1188, https://doi.org/ 10.1175/JTECH-D-14-00019.1.

McGillicuddy, D. J., and Coauthors, 2007: Eddy/wind interactions stimulate extraordinary mid-ocean plankton blooms. Science, 316, 1021-1026, https://doi.org/10.1126/science.1136256.

_ J. R. Ledwell, and L. A. Anderson, 2008: Response to comment on "Eddy/wind interactions stimulate extraordinary midocean plankton blooms." Science, 320, 448, https://doi.org/ 10.1126/science.1148974.

McWilliams, J., 2017: Submesoscale surface fronts and filaments: Secondary circulation, buoyancy flux, and frontogenesis. J. Fluid Mech., 823, 391-432, https://doi.org/10.1017/jfm.2017.294.

_ , J. Gula, J. Molemaker, L. Renault, and A. F. Shchepetkin, 2015: Filament frontogenesis by boundary layer turbulence. J. Phys. Oceanogr., 45, 1988-2005, https://doi.org/10.1175/ JPO-D-14-0211.1.

Mensa, J. A., Z. Garraffo, A. Griffa, T. M. Özgökmen, A. Haza, and M. Veneziani, 2013: Seasonality of the submesoscale dynamics in the Gulf Stream region. Ocean Dyn., 63, 923-941, https://doi.org/10.1007/s10236-013-0633-1.

Nagai, T., A. Tandon, and D. L. Rudnick, 2006: Two-dimensional ageostrophic secondary circulation at ocean fronts due to vertical mixing and large-scale deformation. J. Geophys. Res., 111, C09038, https://doi.org/10.1029/2005JC002964.

Nardelli, B. B., 2013: Vortex waves and vertical motion in a mesoscale cyclonic eddy. J. Geophys. Res. Oceans, 118 , 5609-5624, https://doi.org/10.1002/jgrc.20345.

Niiler, P. P., 1969: On the Ekman divergence in an oceanic jet. J. Geophys. Res., 74, 7048-7052, https://doi.org/10.1029/ JC074i028p07048.

Ohlmann, J. C., M. J. Molemaker, B. Baschek, B. Holt, G. Marmorino, and G. Smith, 2017: Drifter observations of submesoscale flow kinematics in the coastal ocean. Geophys. Res. Lett., 44, 330-337, https://doi.org/10.1002/ 2016 GL071537.

Pallàs-Sanz, E., and A. Viúdez, 2005: Diagnosing mesoscale vertical velocity from horizontal velocity and density data. J. Phys. Oceanogr., 35, 1744-1762, https://doi.org/10.1175/JPO2784.1.

_ T. M. S. Johnston, and D. L. Rudnick, 2010: Frontal dynamics in a California Current System shallow front: 2. Mesoscale vertical velocity. J. Geophys. Res., 115, C12068, https://doi.org/ 10.1029/2010JC006474.

Piedeleu, M., P. Sangrà, A. Sánchez-Vidal, J. Fabrés, C. Gordo, and A. Calafat, 2009: An observational study of oceanic eddy generation mechanisms by tall deep-water islands (Gran Canaria). Geophys. Res. Lett., 36, L14605, https://doi.org/ 10.1029/2008GL037010.

Ponte, A., P. Klein, X. Capet, P. Le Traon, Y. C. Bertrand, and P. Lherminier, 2013: Diagnosing surface mixed layer dynamics from high-resolution satellite observations: Numerical 
insights. J. Phys. Oceanogr., 43, 1345-1355, https://doi.org/ 10.1175/JPO-D-12-0136.1.

Sangrà, P., and Coauthors, 2005: Life history of an anticyclonic eddy. J. Geophys. Res., 110, C03021, https://doi.org/10.1029/ 2004JC002526.

- , and Coauthors, 2007: On the nature of oceanic eddies shed by the island of Gran Canaria. Deep-Sea Res. I, 54, 687-709, https://doi.org/10.1016/j.dsr.2007.02.004.

—_, and Coauthors, 2009: The Canary Eddy Corridor: A major pathway for long-lived eddies in the subtropical North Atlantic. Deep-Sea Res. I, 56, 2100-2114, https://doi.org/ 10.1016/j.dsr.2009.08.008.

Shchepetkin, A. F., and J. C. McWilliams, 2005: The Regional Oceanic Modeling System (ROMS): A split-explicit, freesurface, topography-following-coordinate oceanic model. Ocean Modell., 9, 347-404, https://doi.org/10.1016/j.ocemod.2004.08.002. , and - 2009: Correction and commentary for "Ocean forecasting in terrain-following coordinates: Formulation and skill assessment of the regional ocean modeling system" by Haidvogel et al. J. Comp. Phys. 227, pp. 3595-3624. J. Comput Phys., 228, 8985-9000, https://doi.org/10.1016/j.jcp.2009.09.002.

Sheen, K. L., and Coauthors, 2014: Eddy-induced variability in Southern Ocean abyssal mixing on climatic timescales. Nat. Geosci., 7, 577-582, https://doi.org/10.1038/ngeo2200.

Stern, M., 1965: Interaction of a uniform wind stress with a geostrophic vortex. Deep-Sea Res., 12, 355-367, https://doi.org/ 10.1016/0011-7471(65)90007-0.

Thomas, L. N., and P. B. Rhines, 2002: Nonlinear stratified spinup. J. Fluid Mech., 473, 211-244, https://doi.org/10.1017/ S0022112002002367.
— and C. M. Lee, 2005: Intensification of ocean fronts by downfront winds. J. Phys. Oceanogr., 35, 1086-1102, https://doi.org/ 10.1175/JPO2737.1.

Vallis, G. K., 2006: Atmospheric and Oceanic Fluid Dynamics. Cambridge University Press, 745 pp.

Viúdez, A., 2017: Isolated marginally stable geophysical vortices. J. Fluid Mech., 824, R4, https://doi.org/10.1017/jfm.2017.440. , and D. G. Dritschel, 2003: Vertical velocity in mesoscale geophysical flows. J. Fluid Mech., 483, 199-223, https://doi.org/ 10.1017/S0022112003004191.

— and _ 2004: Potential vorticity and the quasigeostrophic and semigeostrophic mesoscale vertical velocity. J. Phys. Oceanogr., 34, 865-887, https://doi.org/10.1175/1520-0485 (2004)034<0865:PVATQA > 2.0.CO;2.

_ _ J. Tintoré, and R. L. Haney, 1996: About the nature of the generalized omega equation. J. Atmos. Sci., 53, 787-795, https://doi.org/10.1175/1520-0469(1996)053<0787:ATNOTG> 2.0.CO;2.

Wenegrat, J. O., and L. N. Thomas, 2017: Ekman transport in balanced currents with curvature. J. Phys. Oceanogr., 47, 1189-1203, https://doi.org/10.1175/JPO-D-16-0239.1.

Yelland, M., B. I. Moat, P. K. Taylor, R. W. Pascal, J. Hutchings, and V. C. Cornell, 1998: Wind stress measurements from the open ocean corrected for airflow distortion by the ship. J. Phys. Oceanogr., 28, 1511-1526, https://doi.org/10.1175/ 1520-0485(1998)028<1511:WSMFTO > 2.0.CO;2.

Zhong, Y., A. Bracco, and T. Villareal, 2012: Pattern formation at the ocean surface: Sargassum distribution and the role of the eddy field. Limnol. Oceanogr. Fluids Environ., 2, 12-27, https:// doi.org/10.1215/21573689-1573372. 